\title{
Memories of Vladimir Arnold
}

\author{
Boris Khesin and Serge Tabachnikov, \\ Coordinating Editors
}

Vladimir Arnold, an eminent mathematician of our time, passed away on June 3, 2010, nine days before his seventy-third birthday. This article, along with one in the previous issue of the Notices, touches on his outstanding personality and his great contribution to mathematics.

\section{Dmitry Fuchs}

\section{Dima Arnold in My Life}

Unfortunately, I have never been Arnold's student, although as a mathematician, I owe him a lot. He was just two years older than I, and according to the University records, the time distance between us was still less: when I was admitted to the Moscow State university as a freshman, he was a sophomore. We knew each other but did not communicate much. Once, I invited him to participate in a ski hiking trip (we used to travel during the winter breaks in the almost unpopulated northern Russia), but he said that Kolmogorov wanted him to stay in Moscow during the break: they were going to work together. I decided that he was arrogant and never repeated the invitation.

Then he became very famous. Kolmogorov announced that his nineteen year-old student Dima Arnold had completed the solution of Hilbert's 13th problem: every continuous function of three or more variables is a superposition of continuous

Boris Khesin is professor of mathematics at the University of Toronto. His email address is khesin@math. toronto. edu.

Serge Tabachnikov is professor of mathematics at Pennsylvania State University. His email address is tabachni@ math.psu.edu.

Dmitry Fuchs is professor of mathematics at the University of California, Davis. His email address is fuchs@math. ucdavis. edu.

DOI: http://dx.doi.org/10.1090/noti813 functions of two variables. Dima presented a twohour talk at a weekly meeting of the Moscow Mathematical Society; it was very uncommon for the society to have such a young speaker. Everybody ad-

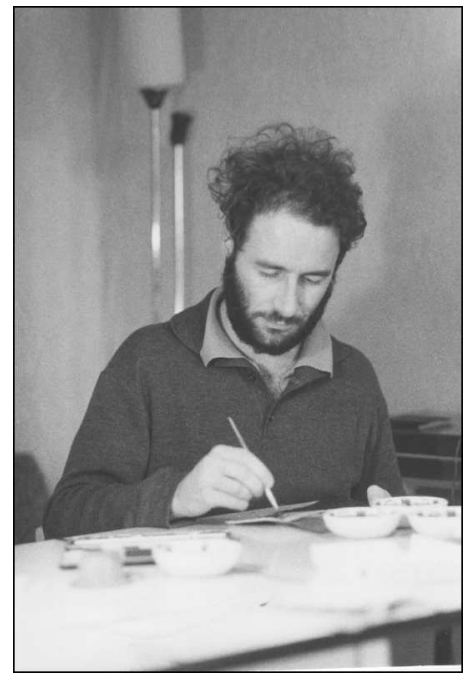

V. Arnold, drawing, 1968. mired him, and he certainly deserved that. Still there was something that kept me at a distance from him.

I belonged to a tiny group of students, led by Sergei Novikov, which studied algebraic topology. Just a decade before, Pontryagin's seminar in Moscow was a true center of the world of topology, but then Cartan's seminar in Paris claimed the leadership, algebraic topology became more algebraic, and the rulers of Moscow mathematics pronounced topology dead. Our friends tried to convince us to drop all these exact sequences and commutative diagrams and do something reasonable, like functional analysis or PDE or probability. However, we were stubborn. We even tried to create something like a topological school, and, already being a graduate student, I delivered a course of lectures in algebraic topology. The lectures were attended by several undergraduates, and we were happy to play this game.

Then something incredible happened. One day I found the lecture room filled beyond capacity; I even had to look for a bigger room. My audience 


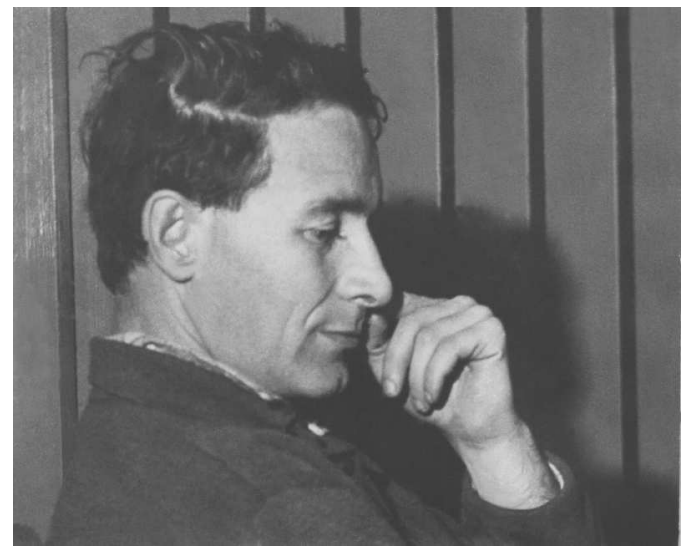

At Otepya, Estonia.

had become diverse: undergraduates, graduate students, professors. This change had a very clear reason: the Atiyah-Singer index theorem.

The problem of finding a topological formula for the index of an elliptic operator belonged to Gelfand. Our PDE people studied indexes a lot, and they had good results. It was not a disaster for them that the final formula was found by somebody else: their works were respectfully cited by Atiyah, Singer, and their followers. The trouble was that the formula stated, "the index is equal to" and then something which they could not understand. People rushed to study topology, and my modest course turned out to be the only place to do that.

And to my great surprise, I noticed Dima Arnold in the crowd.

I must say that Dima never belonged to any crowd. Certainly the reason for his presence did not lie in any particular formula. Simply, he had never dismissed topology as nonsense, but neither had he been aware of my lectures. When he learned of their existence, he appeared. That was all. He never missed a lecture.

One day we met in a long line at the student canteen. "Listen," he said, "can you explain to me what a spectral sequence is?" I began uttering the usual words: a complex, a filtration, differentials, adjoint groups, etc. He frowned and then said, "Thus, there is something invariant ['invariant' in his language meant 'deserving of consideration'] in all this stuff, and this is the spectral sequence, right?" I thought for a moment and said, yes. At this moment we got our meals, and our conversation changed its direction.

Evidently spectral sequences were not for Arnold. Nonetheless, there is such a thing as Arnold's spectral sequence [9], a humble object in the world of his discoveries, resembling the asteroid Vladarnolda in the solar system (the stability of which he proved approximately at the time of our conversation in the canteen), named after him. When I say that he could not appreciate spectral sequences, I mean that he in general had a strong dislike for unnecessary technicalities, and technicalities were often unnecessary to him because of his extremely deep understanding. By the way, this attitude toward impressive but unnecessary tricks extended beyond mathematics. Years later we spent a week or so with friends at a ski resort in Armenia. We showed each other different turns and slidings, but Dima obviously was not interested. He said that the slope was not too steep, and he simply went straight from the top to the bottom, where he somehow managed to stop. I was surprised: there was a stone hedge in the middle of the slope that you needed to go around. Dima said modestly, "You know, at this place my speed is so high that I simply pull my legs up and jump over the hedge." I could not believe it, so I waited at a safe distance from the hedge and watched him doing that. It was more impressive than all our maneuvers taken together. Whatever he did-mathematics, skiing, biking-he preferred not to learn how to do it but just to do it in the most natural way, and he did everything superlatively well.

I do not remember how it came about that I began attending his Tuesday seminar. Probably he asked me to explain some topological work there, then I had to participate in some discussion, and then I could not imagine my life without spending two hours every Tuesday evening in a small room on the fourteenth floor of the main building of the MSU. Works of Arnold, his numerous students, and other selected people were presented at the seminar, and Dima insisted that every word of every talk be clear to everybody in the audience. My role there was well established: I had to resolve any topology-related difficulty. Some of my friends said that at Arnold's seminar I was a "cold topologist". Certainly, a non-Russian-speaker cannot understand this, so let me explain. In many Russian cities there were "cold shoemakers" in the streets who could provide an urgent repair to your footwear. They sat in their booths, usually with no heating (this is why they were "cold"), and shouted, "Heels!...Soles!...." So I appeared as if sitting in a cold booth and yelling, "Cohomology rings!...Homotopy groups!...Characteristic classes!...."

In my capacity as cold topologist, I even had to publish two short articles. One was called "On the Maslov-Arnold characteristic classes," and the other one had an amusing history. One day Dima approached me before a talk at the Moscow Mathematical Society and asked whether I could compute the cohomology of the pure braid group ("colored braid group" in Russian); he needed it urgently. I requested a description of the classifying space, and the calculation was ready at the end of the talk. It turned out that the (integral) cohomology ring was isomorphic 
to a subring of the ring of differential forms on the classifying manifold. He suggested that I write a note, but I refused: for a topologist it was just an exercise; it could be interesting only in conjunction with an application to something else. (I knew that Dima was thinking of Hilbert's 13th problem in its algebraic form: the possibility of solving a general equation of degree 7 not in radicals, but in algebraic functions of two variables.) I suggested that he write an article and mention my modest contribution in an appropriate place. He did [2]. But, a couple of months later, he needed the cohomology of the classical Artin's braid group. This was more difficult and took me several days to complete the calculation. I did it only modulo 2 , but I calculated a full ring structure and also the action of the Steenrod squares. (The integral cohomology was later calculated independently by F. Vainshtein, V. Goryunov, and F. Cohen and still later Graeme Segal proved that the classifying space of the infinite braid group was homologically equivalent to $\Omega^{2} S^{3}$.) I phoned Dima and explained the results. First he requested that I give a talk at the seminar (Next Tuesday! That is tomorrow!), and then he decidedly refused to do what we had agreed upon for pure braids: to write an article and mention my participation where appropriate. After a brief argument, we arrived at a compromise: I publish an article about the cohomology of the braid group without any mentioning of Hilbert's problem, and he publishes an article where this cohomology is applied to superpositions of algebraic functions. When we met the next day, his article was fully written and mine had not even been started. But his article contained a reference to mine and hence the title of the latter. I could delay no longer, and the two articles were published in the same volume of Functional Analysis [5], [14]. Since the articles in Functional Analysis were arranged alphabetically, his article was the first, and mine was the last. But this was not the end of the story. A cover-to-cover translation of Functional Analysis was published by an American publisher. The braid group in Russian is called группа кос; the word кос is simply the genitive of коса, a braid, but the American translators thought that $\mathrm{KOC}$ was a Russian equivalent of COS, and the English translation of my article was attributed to a mysterious cosine group. I do not know how many English-speaking readers of the journal tried to guess what the cosine group was.

As a permanent participant of Arnold's seminar, I had an opportunity to give talks on my works not explicitly related to the main directions of the seminar. I gave a brief account of my work with Gelfand on the cohomology of infinite-dimensional Lie algebras, of characteristic classes of foliations. These things did not interest Dima much, although he himself had a work on

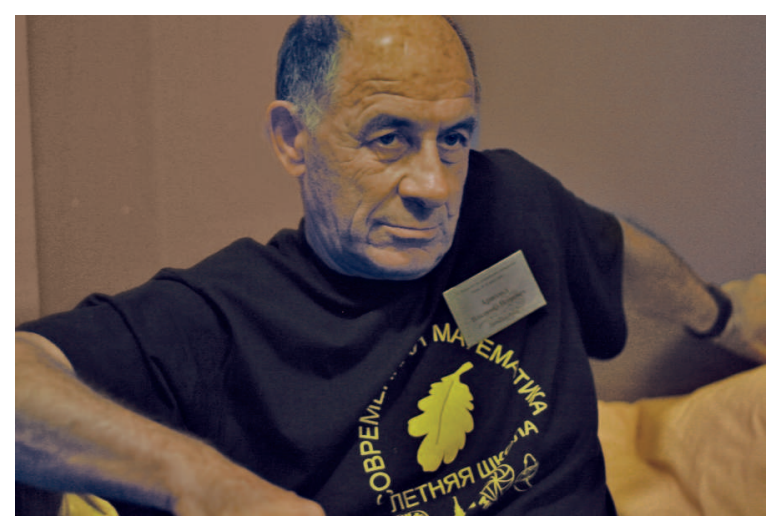

Summer School "Contemporary Mathematics" at Dubna, near Moscow, 2006.

similar things [3]. He always considered algebra and topology as something auxiliary. Once I heard him saying respectfully, "Siegel's case, this is a true analysis," and this sounded like "true mathematics". Whatever he did, his unbelievably deep understanding of analysis was always his main instrument.

One more story of a similar kind. In 1982 John Milnor, who briefly visited Moscow, delivered a talk at Arnold's seminar on a very recent (and not yet published) work of D. Bennequin on a new invariant in the theory of Legendrian knots in contact 3-manifolds. The main result of Bennequin stated that the "Bennequin number" (now justly called the "Thurston-Bennequin number") of a topologically unknotted Legendrian knot in the standard contact space must be strictly negative. For an illustration, Milnor showed an example of a Legendrian trefoil with the Bennequin number +1 . Arnold said that at last he had seen a convincing proof that the trefoil is a topologically nontrivial knot. Certainly, this was a joke: Bennequin's proof at that time did not look convincing, and the nontriviality of the trefoil has a popular proof understandable to middle school students (via the tricolorability invariant). But for Dima only an analytic proof could be fully convincing.

When I joined the Arnold seminar, it had just acquired the name of "the seminar on singularities of smooth maps". In the mid-1960s, Arnold was fascinated by work of John Mather on singularities. People could not understand this. Allegedly, Pontryagin said: "We can always remove complicated singularities of a smooth map by a small perturbation; it is sufficient to study the generic case." But singularities appear in families of smooth maps; you cannot remove them, insisted Dima. Some people mocked his affection for singularity theory. There is a short story of Stanislav Lem (a Polish science fiction writer) in which robots that could experience human emotions were manufactured. One of these robots felt an immense joy when he solved quadratic equations-just like you, Dima! 
Dima smiled at such jokes but continued studying singularities.

The results of Arnold and his students in this area were very deep and diverse. He classified all singularities that appear in generic families depending on no more than 14 parameters and studied their moduli varieties and discriminants. He discovered the relations of the theory to symplectic, contact, and differential geometry. It had deep applications in topology (Vassiliev's invariants of knots), differential equations, and classical mechanics.

More or less at the same time, a widely popularized version of the singularity theory emerged under the colorful name of the theory of catastrophes. It was promoted by two remarkable topologists, R. Thom and E. C. Zeeman. "The most catastrophic feature of the theory of catastrophes is a full absence of references to the works of H. Whitney," Dima wrote in one of his books. Indeed, mathematically, the theory of catastrophes was based on a classification of singularities of generic smooth maps of a plane onto a plane. The classification was fully done in 1955 by Whitney [15], but the founding fathers of catastrophe theory preferred to pretend that the works of Whitney never existed. Still, Dima made his contribution to the popularization of catastrophes: he wrote a short popular book under the title "Theory of Catastrophes". It was written in 1983 and then translated into a dozen languages.

In 1990 I moved to a different country, and we met only four or five times after that. The last time that I saw him was in spring 2007, when he visited California. We travelled together through the Napa and Sonoma Valleys; he was especially interested in visiting Jack London's grave. He spoke endlessly of his new (was it new?) passion for continued fractions, numerical functions, and numerical experimentation. I boasted that I taught a course of history of mathematics, and he immediately began testing my knowledge of the subject: Who proved the Euler theorem of polyhedra? Who proved the Stokes theorem? To his apparent displeasure I passed the exam. (He was especially surprised that I knew that Descartes proved the Euler theorem more than one hundred years before Euler. Why do you know that? I said that Efremovich told me this some thirty years before.) More than that, I knew something that he did not know: the Stokes theorem as it is stated in modern books, $\int_{C} d \varphi=\int_{\partial C} \varphi$, was first proved and published by the French mathematician E. Goursat (1917). We discussed a bit our further plans, and Dima said that whatever he plans, he always adds, as Leo Tolstoy did, ЕБЖ = если буду жив, "If I am alive." I said that I also never forget to add this, but apparently neither of us took it seriously. Anyhow, we never met again.
My tale of Dima Arnold is becoming lengthy, although I feel that what I have said is a small fraction of what I could say about this tremendous personality. Still, the story would be incomplete if I did not mention something known to everybody who has ever communicated with him, if only occasionally: his universal knowledge of everything. Whatever the subject was-Chinese history, African geography, French literature, the sky full of stars (especially this: he could speak endlessly

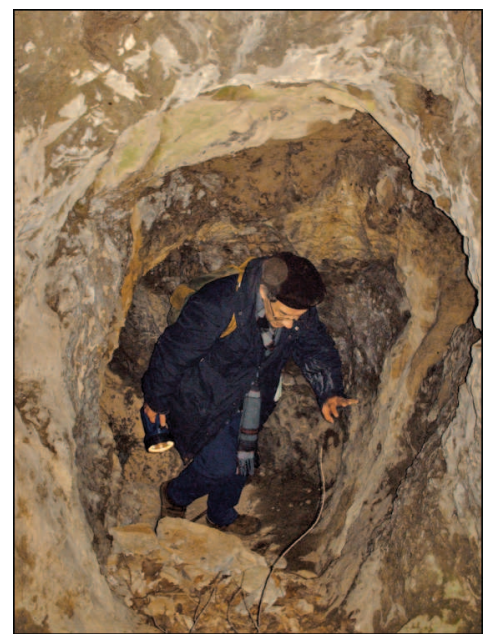

In a cavern, 2008. on every star in every constellation)-he demonstrated without effort a familiarity with the subject which exceeded and dwarfed everybody else's, and this, combined with his natural talent as a storyteller, made every meeting with him a memorable event. Some friends recollect a sight-seeing tour in Paris he gave a couple of months before his death. Obviously, no tourist agency ever had a guide of this quality. Instead of adding my own recollections, I finish my account with a translation of a letter I received from him a year after our last meeting and two years before his death.

$$
\begin{aligned}
& \text { Paris, March 26, } 2008 \\
& \text { Dear Mitya, } \\
& \text { I have recently returned to }
\end{aligned}
$$
Paris from Italy where I wandered, for three months, in karstic mountains working at ICPT (the International Center for Theoretical Physics) at Miramare, the estate of the Austrian prince Maximilian who was persuaded by Napoleon III to become the Emperor of Mexico (for which he was shot around 1867 as shown in the famous and blood-drenched picture of Edouard Manet).

I lived in the village of Sistiana, some 10 kilometers from Miramare in the direction of Venice. It was founded by the pope Sixtus, the same one who gave names to both the chapel and Madonna. Passing the POKOPALIŠCE ${ }^{1}$ (the cemetery) some 3 versts $^{2}$ to the North, I reached a deer path in a mountain pine grove. These deer do

\footnotetext{
${ }^{1}$ This word has a notable similarity to Russian KOПАТЬ, to dig.

${ }^{2}$ BEPCTA is an old Russian measure of length, $\approx 1.1 \mathrm{~km}$.
} 
not pay much attention to a small tin sign, DERŽAVNAYA MEŽA ${ }^{3}$ (the state border). After that it is Slovenia to which I ran, following the deer. But at the next sign, PERICOLO, the deer refused to go any farther. The local people (whose language is closer to Russian than Ukrainian or Bulgarian) explained to me that the sign is a warning that the nearby caves have not been demined. And they were mined during the FIRST world war when my deer path was called SENTIERA DIGUERRA and was a front line (described by Hemingway in "A Farewell to Arms").

I did not go down to these particular caves, but every day I visited tens of them, of which some (but not all) were shown on a map (where they were called YAMA, ${ }^{4}$ GROTTA, CAVA, CAVERNA, ABISSA, dependingly of the difficulty of the descent). All these caves look pretty much the same (a colorful scheme is provided): there is a hole on the mountain, a meter in size, and down go walls, of not even vertical but rather a negative slope. The depth of the mine is usually around 10 or 20 meters (but I descended to YAMA FIGOVICHEVA with the officially declared depth of 24 meters and to the half of the height, or rather the depth, of GROTTA TERNOVIZZA whose depth is marked as 32 meters and to which one cannot descend without a rope). At the bottom of the YAMA a diverging labyrinth of passages starts, of the lengths on the order of 100 meters. They go to lakes, stalactites, etc. Sometimes there is even a descent to the Timavo river (which flows about 50 kilometers at the depth 100 or 200 meters, depending on the height of hills above). Before this 50 kilometers it is a forest river resembling Moscow River at Nikolina Gora ${ }^{5}$ with a charming Roman name of REKA. ${ }^{6}$

\footnotetext{
${ }^{3}$ Both words belong to old Russian.

${ }^{4}$ Russian AMA means a gap.

${ }^{5}$ A village some 30 kilometers from Moscow where many remarkable Russian people (including Dima) used to spend their vacations.

${ }^{6}$ PEKA is the Russian for a river.
}

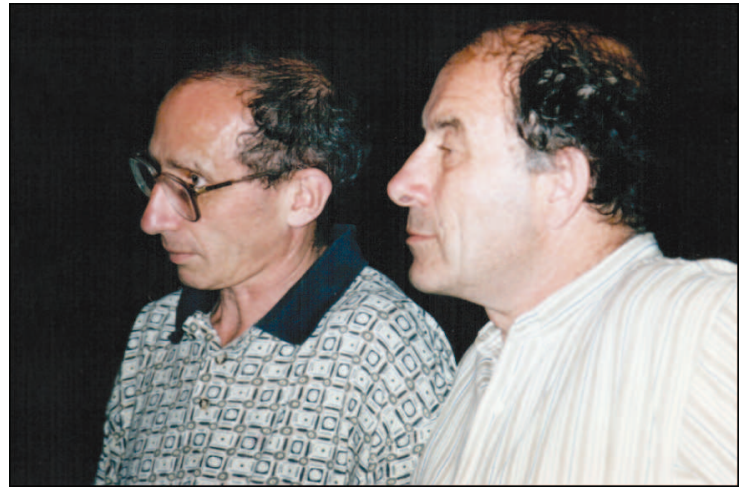

Ya. Eliashberg and V. Arnold, 1997.

This was a part of Jason's expedition (with argonauts). On his way back from Colchis (with the golden fleece) he sailed his ship Argo upstream Ister (Danube) and its tributaries to the Croatian peninsula named Capudistria (which is visible from my window at Sistiana), then they dragged the ship to REKA and, following Timavo, they reached to northernmost point of the Adriatic, where the Roman city of Aquileia was later built.

Near Aquileia, I discovered a goddess Methe, new to me, but this is a separate story. (She saves any drinker of drunkenness, however much he drank. Allegedly, she was the mother of Athena, and Jupiter ate her, since he was afraid that she would give birth to a son, and that this son would dethrone him, precisely as he himself had dethroned his father.) Aquileia is a Roman port of the first century, preserved as well as Pompeii, without any Vesuvius: simply Attila who destroyed the city left the port intact, including the canals, ships (which survive to our time), quays, knechts and basilicas (which became Christian in the IV century) with mosaics of $50 \mathrm{~m} \times 100 \mathrm{~m}$ in size, and absolutely everything as in Pompeii. No room to describe everything, I am just sending my best (Easter) wishes.

On June 3, I go to Moscow, there will be a conference dedicated to the centenary of LSP. ${ }^{7}$

Dima

\footnotetext{
${ }^{7}$ Lev Semenovich Pontryagin
} 


\section{Yakov Eliashberg}

\section{My Encounters with Vladimir Igorevich Arnold}

My formation as a mathematician was greatly influenced by Vladimir Igorevich Arnold, though I never was his student and even lived in a different city. When I entered Leningrad University in 1964 as an undergraduate math student, Arnold was already a famous mathematician. By that time he had solved Hilbert's 13th problem and had written a series of papers which made him the "A" in the KAM theory. Arnold was also working as an editor of the publishing house Mir, where he organized and edited translations of several books and collections of papers not readily accessible in the USSR. One of these books, a collection of papers on singularities of differentiable mappings, was an eye opener for me.

The first time I met Arnold was in January 1969 at a Winter Mathematical School at Tsakhkadzor in Armenia. I was eager to tell him about some of my recently proved results concerning the topology of singularities. Later that year he invited me to give a talk at his famous Moscow seminar. I remember being extremely nervous going there. I could not sleep at all in the night train from Leningrad to Moscow, and I do not remember anything about the talk itself.

In 1972 Vladimir Igorevich was one of my Ph.D. dissertation referees or, as it was called, an "official opponent". I remember that on the day of my defense, I met him at 5 a.m. at the Moscow Train Station in Leningrad. He immediately told me that one of the lemmas in my thesis was wrong. It was a local lemma about the normal form of singularities, and I thought (and, frankly, still do) that the claim is obvious. I spent the next two hours trying to convince Vladimir Igorevich, and he finally conceded that probably the claim is correct, but still insisted that I did not really have the proof. A year later he wrote a paper devoted to the proof of that lemma and sent me a preprint with a note that now my dissertation is on firm ground.

After my Ph.D. defense I was sent to work at a newly organized university in Syktyvkar, the capital of Komi Republic in the north of Russia. In 1977 we organized there a conference on global analysis which attracted a stellar list of participants, including V. I. Arnold. During this conference I asked Arnold to give a lecture for our undergraduate students. He readily agreed and gave an extremely interesting lecture about stability of the inverse pendulum, and even made a demonstration prepared with the help of one of

Yakov Eliashberg is professor of mathematics at Stanford University. His email address is eliash@math. stanford.edu.

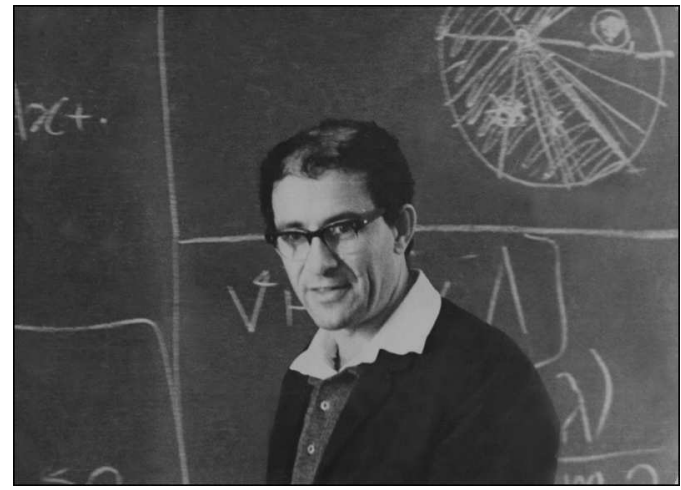

A talk at Syktyvkar, 1976.

our professors, Alesha Zhubr. Arnold had certain pedagogical methods to keep the audience awake. During his lectures he liked to make small mistakes, expecting students to notice and correct him. Apparently, this method worked quite well at the Moscow University. Following the same routine during his Syktyvkar lecture, he made an obvious computational error-something like forgetting the minus sign in the formula $(\cos x)^{\prime}=-\sin x-$ and expected somebody in the audience to correct him. No one did, and he had to continue with the computation, which, of course, went astray: the terms which were supposed to cancel did not. Very irritated, Arnold erased the blackboard and started the computation all over again, this time without any mistakes. After the lecture, he told me that the undergraduate students at Syktyvkar University are very bad. The next day, after my regular class, a few students came to me and asked how is it possible that such a famous mathematician is making mistakes in differentiating $\cos x$ ?

Whenever I happened to be in Moscow, which was not very often, Arnold usually invited me to visit the hospitable home he shared with his wife, Elya. When he moved to a new apartment in Yasenevo on the outskirts of Moscow, he told me over the phone how to get there. In particular, I was instructed to walk south when I got out of the metro station. When I got to that point it was a dark gray late winter afternoon, and it was quite a challenge to figure out in which direction I should go.

Once he ran a psychological test on me to determine which of my brain hemispheres is the dominant one. To his satisfaction, the test showed that it was the right one, which, according to Arnold, meant that I have a geometric rather than an algebraic way of thinking. During another visit, I was deeply honored when he told me that while he files most preprints systematically, I was among the few people who were assigned a personal folder.

Over the years I gave a number of talks at his seminar with variable success. The most disastrous was my last talk in 1985. Shortly before one 
of my trips to Moscow, Misha Gromov sent me a preliminary version of his now very famous paper "Pseudoholomorphic curves in symplectic geometry", which is one of the major foundational milestones of symplectic topology. I was extremely excited about this paper and thus volunteered to talk about it at Arnold's seminar. I think that I was at this moment the only person in the Soviet Union who had the paper. Arnold heard about Gromov's breakthrough but had not seen the paper yet. After a few minutes of my talk, Arnold interrupted me and requested that before continuing I should explain what is the main idea of the paper. This paper is full of new ideas and, in my opinion, it is quite subjective to say which one is the main one. I made several attempts to start from different points, but Arnold was never satisfied. Finally, towards the end of the two-hour long seminar, I said something which Arnold liked. "Why did you waste our time and did not start with this from the very beginning?", he demanded.

Vladimir Igorevich made two long visits to Stanford. During his first quarter-long visit Arnold was giving a lecture course, but he made it a rule for himself to go every morning for a long bike ride into the hills (called the Santa Cruz Mountains) surrounding Stanford. I have heard a lot of stories about Arnold's superhuman endurance and his extremely risky adventures, especially in his younger years. I can testify that at almost sixty years old, Arnold at Stanford was also very impressive. On a windy day after swimming in our cold Pacific Ocean, where the water temperature is usually around $13^{\circ} \mathrm{C}$, he refused a towel. He had a very poor bike which was not especially suited for mountain biking. Yet he went with it everywhere, even over the roads whose parts were destroyed by a mudslide and where he had to climb clutching the tree roots, hauling his bike on his back. During one of these trips, Vladimir Igorevich met a mountain lion. He described this encounter in one of his short stories. Both Arnold and the lion were apparently equally impressed with the meeting. Many years later, during his second visit to Stanford, Arnold again went to the same place hoping to meet the mountain lion. Amazingly, the lion waited for him there! I am also fond of hiking in those hills, yet neither I nor any of people I know ever met a mountain lion there.

When he was leaving Stanford, Vladimir Igorevich gave me a present-a map of the local hills on which he had marked several interesting places that he had discovered, such as an abandoned apple farm or a walnut tree grove.

In between the two visits Arnold had a terrible bike accident in Paris which he barely survived. It was a great relief to see him active again when I met him in Paris two years later. He proudly told me that during this year he had written five books. "One of these books," he said, "is coauthored with

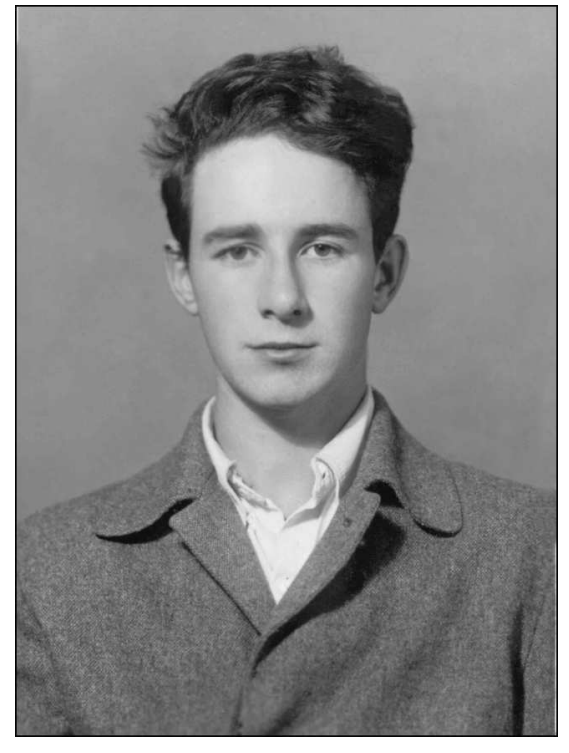

Vladimir Arnold, 1957.

two presidents. Can you guess with which ones?" I certainly could not guess that these were Vladimir Putin and George W. Bush.

During his last visit to Stanford and Berkeley a year ago, Arnold gave two series of lectures: one for "Stanford professors", as he called it, and the other for the school-age children at Berkeley Math Circles. There is no telling which of these two groups of listeners Vladimir Igorevich preferred. He spent all his time preparing for his lectures for children and even wrote a book for them. Lectures at Stanford were an obvious distraction from that main activity. Each Stanford lecture he would usually start with a sentence like "What I am going to talk about now is known to most kindergarten children in Moscow, but for Stanford professors I do need to explain this." What followed was always fascinating and very interesting.

It is hard to come to terms that Vladimir Igorevich Arnold is no longer with us. It is certainly true, though commonplace to say, that Arnold was a great and extremely influential mathematician, that he created several mathematical schools, and that his vision and conjectures shaped a large part of modern mathematics. But, besides all that, he was a catalyst for the mathematical community. He hated and always fought mediocrity everywhere. With his extreme and sometimes intentionally outrageous claims, he kept everybody on guard, not allowing us to comfortably fall asleep.

His departure is also painful to me because there are several unfulfilled mathematical promises which I made to him but never had time to finish. Though it is too late, I will do it now as a priority. 


\section{Yulij Ilyashenko}

\section{I. Arnold, As I Have Seen Him}

A student, visiting his schoolmaster in math, the famous and severe Morozkin. A radiant slim youth, almost a boy. This was Arnold as I first saw him, more than fifty years ago.

A graduate student (in 1960), conducting tutorials in honors calculus (taught to freshmen at Mekhmat, the Department of Mechanics and Mathematics of the Moscow State University). There was a permanent kind of smile on his face, his eyes were sparkling, and when he looked at you, a wave of good will would come forth.

From 1968 to 1986 I had the privilege of working with Arnold at the same section of Mekhmat, called the "Division of Differential Equations". It was shaped by Petrovski and chaired by him until his premature death in 1973. When Arnold joined the division, it was full of the best experts in differential equations, partial and ordinary. Besides Arnold and Petrovski, the faculty of the division included stars of the elder generation (who were then in their thirties and forties): Landis, Oleinik, Vishik, as well as brilliant mathematicians of Arnold's generation: Egorov, Kondratiev, Kruzhkov, and others.

The first glorious results of Arnold are described in other papers in this collection. Let me turn to differential equations, a subject whose development I have been closely following. Needless to say, these are personal remarks, not a complete history.

In 1965 Arnold came back from France, where he spent almost a year. From there he brought a keen interest in the newborn singularity theory, of which he became one of the founding fathers. He also brought the philosophy of general position invented by René Thom, which became sort of a compass in Arnold's investigations in differential equations and bifurcation theory.

In the form that Arnold gave to it, this philosophy claimed that one should first investigate objects in general position, then the simplest degenerations, together with their unfoldings. It makes no sense to study degenerations of higher codimension until those of smaller codimension have been investigated.

In 1970 he published a short paper [6], in which a strategy for developing any kind of local theory based on the above philosophy was suggested. He also defined algebraically solvable local problems. He started to call them "trivial", but later stopped doing that. "Let us forget the overloaded term," he once told me about this word. In the same paper he also stated that the problem of distinguishing

Yulij Ilyashenko is professor of mathematics at Cornell University and Independent University of Moscow. His email address is yu1ijs@gmai 1.com. center and focus is trivial. Bruno challenged this statement, and I proved that the center-focus problem is algebraically unsolvable (1972).

Also in 1970 Arnold proved that the problem of Lyapunov stability is algebraically unsolvable. He constructed a 3-parameter family in the space of high-order jets, where the boundary of stability is nonalgebraic. In the same paper he wrote: "One may expect that the Lyapunov stability, having lost algebraicity and no more restricted by anything, may present some pathologies on the set theoretic-level..." He also suggested that the problem may be algorithmically unsolvable. This conjecture is still open. In the mid-1970s it turned out that a nonalgebraic boundary of Lyapunov stability occurs in unfoldings of degenerations of codimension three in the phase spaces of dimension four. This was discovered by Shnol' and Khazin, who investigated the stability problem in the spirit of Arnold and studied all the degenerate cases up to codimension three.

In 1969 Bruno defended his famous doctoral thesis about analytic normal forms of differential equations near singular points. One of his results is the so-called Bruno condition: a sufficient condition for the germ of a map to be analytically equivalent to its linear part. In dimension one, Yoccoz proved the necessity of this condition (1987); this result was rewarded by a Fields Medal, which he got in 1994. So the problem is still a focus of interest in the mathematics community. But let us get back to the late 1960s. In his review of the Bruno thesis, Arnold wrote: "The existing proofs of the divergence [of normalizing series] are based on computations of the growth of coefficients and do not explain its nature (in the same sense as the computation of the coefficients of the series $\arctan z$ does not explain the divergence of this series for $|z|>1$, although it proves this divergence)." Following this idea, Arnold tried to find a geometric explanation of the divergence of normalizing series when the denominators are too small. He predicted an effect which he later called "materialization of resonances". An "almost resonant" germ of a vector field that gives rise to "exceedingly small denominators" is close to a countable number of resonant germs. Under the unfolding of any such germ, an invariant manifold bifurcates from a union of coordinate planes and remains in a small neighborhood of the singular point of this almost resonant germ. These invariant manifolds, which constitute a countable number of "materialized resonances", accumulate to the singular point and prevent the linearization.

A. Pyartli, a student of Arnold, justified this heuristic description in his thesis in the early $1970 \mathrm{~s}$ for vector fields with planar saddles. He continued the investigation and in 1976 found an invariant cylinder, a materialization of resonances for a germ of a planar map. Then he asked Arnold, "Why does 


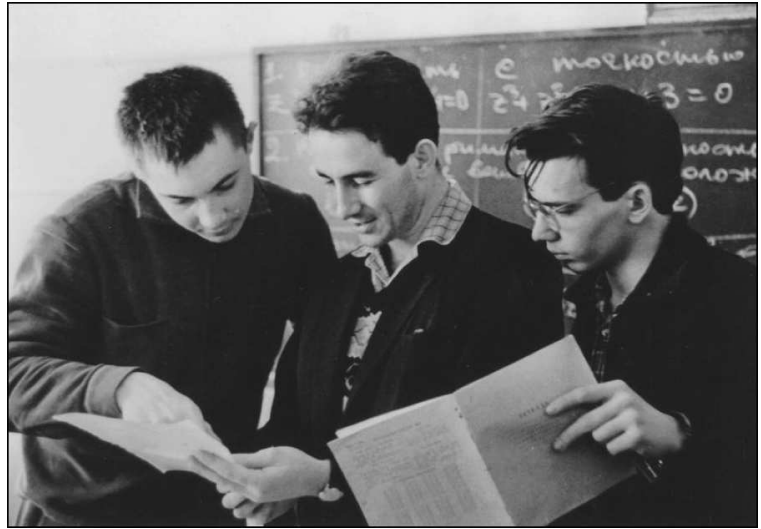

With students of Moscow Mathematics Boarding School, 1960s.

such a cylinder prevent the linearization?" Why, indeed?! Arnold himself started thinking about the problem and came to the theory of normal forms for neighborhoods of embedded elliptic curves. An overview of this theory is given in his book [12]. As usual, this new path was paved by the followers of Arnold: Pyartli, myself, Saveliev, Sedykh, and others.

Arnold's approach to the local bifurcation theory produced a genuine revolution. In the late 1960s he suggested to his students two problems: to prove a reduction principle that excludes excessive "hyperbolic variables" from any local bifurcation problem and to study the first really difficult bifurcation problem in codimension two. The first problem was solved by A. Shoshitaishvili, the second one by R. Bogdanov. "It was not by chance that I launched two different people in two directions simultaneously," Arnold later said to me. Arnold was especially proud that Bogdanov proved the uniqueness of the limit cycle that occurs under the perturbation of a generic cuspidal singular point. F. Takens investigated independently the same codimension two bifurcation as Bogdanov; it is now named the "Bogdanov-Takens" bifurcation.

In [8] Arnold described the new approach to the theory and listed all problems that occur in the study of local bifurcations of singular points of vector fields in codimension two. This was a long-standing program. J. Guckenheimer and N. Gavrilov made important contributions to its development; final solutions were obtained by H. Zoladec (in the mid-1980s), again under the (nonofficial) supervision of Arnold.

In the mid-1970s Arnold himself considered another local bifurcation problem in codimension two, the one for periodic orbits. He discovered strong resonances in the problem and predicted all possible unfoldings occurring in generic perturbations of the Poincaré maps with these resonances (1977). There were four of them. The first case was reduced to Bogdanov-Takens; two other cases were investigated by E. Horosov (1979), a graduate student of Arnold, in his Ph.D. thesis. The fourth case, the famous resonance $1: 4$, was investigated by A. Neishtadt, F. Berezovskaya, A. Khibnik (influenced by Arnold), and B. Krauskopf, a student of Takens. The problem that remains unsolved for bifurcations of codimension two is the existence of very narrow chaotic domains in the parameter and phase spaces.

Later local bifurcations of codimension three were investigated by Dumortier, Roussarie, Sotomayor, and others. The bifurcation diagrams and the phase portraits became more and more complicated. It became clear that it is hopeless to get a complete picture in codimension four. The new part of the bifurcation theory started by Arnold and his school seems to be completed by now. What is described above is a very small part of the new domains that were opened in mathematics by Arnold.

One should not forget that Arnold also inspired many discoveries in oral communications, while no trace of this influence is left in his publications. For instance, he discovered "hidden dynamics" in various problems of singularity theory. This means that a classification problem for singularities often gives rise, in a nonevident way, to a classification problem for special local maps. Thus, he inspired the solution by S. Voronin (1982) of the local classification problem for singularities of envelopes for families of planar curves and the discovery of quite unexpected Ecalle-Voronin moduli of the analytic classification of parabolic fixed points (1981).

Arnold suggested a sketch of the proof of analytic unsolvability of the Lyapunov stability problem (Ilyashenko, 1976). Only later did I understand that, honestly speaking, it should have been a joint work.

In 1980 he pointed out that our joint work with A. Chetaev on an estimate of the Hausdorff dimension of attractors might be applied to the 2D Navier-Stokes equation. This gave rise to an explicit estimate of the Hausdorff dimension of these attractors (Ilyashenko, 1982-83), a first step in the subject later developed by O. Ladyzhenskaya and M. Vishik with his school.

This is only my personal experience, a minor part of the great panorama of Arnold's influence on contemporary mathematics. He had a very strong feeling of mathematical beauty, and his mathematics was at the same time poetry and art. From my youth, I considered Arnold as a Pushkin in mathematics. At present, Pushkin is a beloved treasure of the Russian culture, but during his life, he was not at all treated as a treasure. 


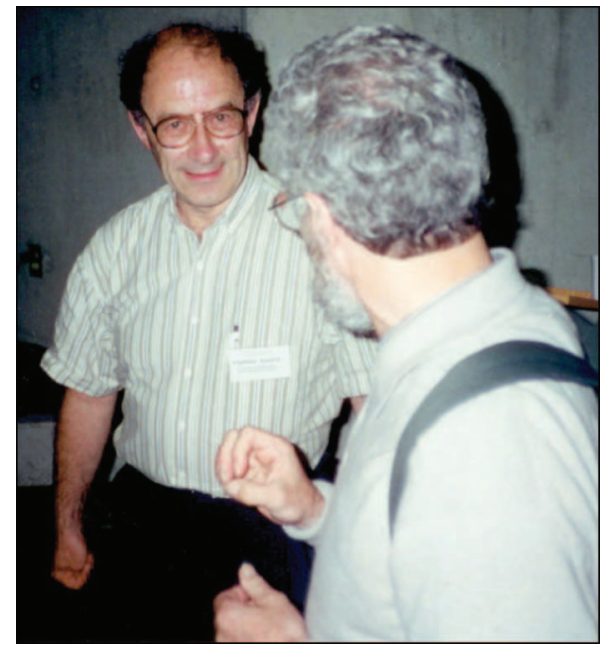

V. Arnold and Yu. Ilyashenko, 1997.

The same is true for Arnold. His life in Russia before perestroika was in no way a bed of roses. I remember very well how we young admirers of Arnold expected in 1974 that he would be awarded the Fields Medal at the ICM at Vancouver. He did not receive it, and the rumor was that Pontryagin, the head of the Soviet National Mathematics Committee, at the discussion of the future awards said, "I do not know the works of such a mathematician." For sure, it could not have been the personal attitude of Pontryagin only; it was actually the position of the Soviet government itself. Two medals instead of four were awarded that year. Much later, Arnold wrote that one of the others was intended for him, and then awarded to nobody.

In 1984 a very skillful baiting of Arnold was organized at Mekhmat. As a result, he had a serious hypertension attack. His election as a corresponding member of the Soviet Academy of Sciences stopped the baiting, but his enemies tried (though unsuccessfully) to renew it five years later.

In 1986 Arnold decided to quit Mekhmat and to move to the Steklov Institute. Yet he wanted to keep a half-time position of professor at Mekhmat. Only after considerable efforts did he get the desired half-time position. I tried to convince Arnold not to quit Mekhmat. I asked him, "Dima, who may say, following Louis XIV's 'L'etat s'est moi,' Mekhmat is me?" "Well," he answered, "I guess NN" (he named an influential party member at the department). "No, Dima, YOU are Mekhmat." But he did not listen.

In 1994 he quit Mekhmat completely. He was offended. He taught a course and a seminar, and suddenly he was informed that this load was insufficient for the half-time position of professor, but only for a quarter-time position (a status that does not, in fact, exist). He spoke with the head of Mekhmat Human Resources. This was an aged woman who maintained her position from the communist times. "She screamed at me," said Arnold with a sort of surprise. Then he resigned from the Moscow State University.

Needless to say, in such an environment the students of Arnold were not hired at Mekhmat. The only exceptions were N. Nekhoroshev and A. Koushnirenko, hired in the early 1970s, and much later A. Varchenko. I remember two other attempts, both unsuccessful. At the same time, the best of the best Mekhmat students asked Arnold to be their advisor. So, Mekhmat rejected the best of the best of its alumni. The same happened with students of Manin, Kirillov, Gelfand....At the end of the 1980s, a critical mass of excellent mathematicians not involved in the official academic life had accumulated. Following a suggestion of N. N. Konstantinov, a well-known educator and organizer of mathematical olympiads, these mathematicians decided to create their own university. In 1991 a group of leading Russian mathematicians formed a council and established a new Independent University of Moscow, IUM. This group included the following members of the Russian Academy of Sciences: V. I. Arnold (chairman of the council), S. P. Novikov, Ya. G. Sinai, L. D. Faddeev; and the following professors: A. A. Beilinson, R. L. Dobrushin, B. A. Dubrovin, A. A. Kirillov, A. N. Rudakov, V. M. Tikhomirov, A. G. Khovanskii, M. A. Shubin. Professors P. Deligne and R. MacPherson of Princeton and MIT also played crucial roles in the founding of the Independent University.

Arnold was very enthusiastic about the new university, and in the first years of its existence he did a lot to shape its spirit and teaching style. Together with the first dean of the College of Mathematics of the IUM, A. Rudakov, Arnold thoroughly discussed the programs, and he himself taught a course on partial differential equations. Under his influence, the Independent University became one of the focal centers of Russian mathematical life.

In 1994 another educational institution, the Moscow Center of Continuous Mathematical Education (MCCME), was created. From the very beginning, Arnold was the head of the board of trustees of this center. The center, headed by I. Yashchenko, the director, became a very influential institution in Russian mathematical education and a powerful tool in the struggle against modern obscurantism. Arnold was one of the leaders of that struggle.

In 2005 Pierre Deligne, together with the IUM faculty, organized a contest for young Russian mathematicians. This contest was funded by Deligne from his Balzan Prize (and named after 
him) with the goal "to support Russian mathematics, struggling for survival." The funds of the contest were strictly limited. In 2006 Arnold met D. Zimin, the head of “D. B. Zimin's Charity Foundation Dynasty", and convinced him to establish a similar "Dynasty contest". Now the contest has become permanent, Lord willing and the creek don't rise, as the proverb says. This is only one of the examples of the long-lasting influence of Arnold on Russian mathematical life.

Arnold's talks were always special events. He began giving lectures at Mekhmat in September 1961 about the newborn theory later named KAM (Kolmogorov-Arnold-Moser). A rumor spread among the students that "Arnold has solved problems that Poincaré failed to solve." His lectures were very fast and intense, yet they attracted the best students in the department. He repeated this course twice, in 1962-63 and in 1963-64.

After that he gave brilliant courses in theoretical mechanics, ordinary differential equations, supplementary chapters of ODE, singularity theory, geometric theory of PDE, and many others. All these courses gave rise to world-famous books, written by Arnold, sometimes with his students. In 1968 Arnold started teaching a course in ODE that became, in a sense, a course of his life. He taught it every year until the late eighties, except for sabbaticals.

Arnold completely changed the face of the discipline. His presentation was coordinate-free: all the constructions were invariant with respect to coordinate changes. "When you present material in coordinates," he said, "you study your coordinate system, not the effect that you want to describe." His language was quite different from that of the previous textbooks and courses: diffeomorphisms, phase flows, rectification of vector fields, exponentials of linear operators....The language of pictures was even more important in his course than that of formulas. He always required a student to present the answer in both ways, a formula and a figure, and to explain the relation between them. He drastically renewed the problem sets for the course: propagation of rays in nonuniform media and geodesics on surfaces of revolution, phase portraits of the Newton equation with one degree of freedom, images of the unit square under linear phase flows-students were expected to draft all of these even without explicit calculations of the corresponding solutions. In the first years the course was difficult both for students and teaching assistants. Later on it smoothed out and became one of the highlights of the Mekhmat curriculum.

All his life V. I. Arnold was like a star that shines, sparkles, and produces new life around it.

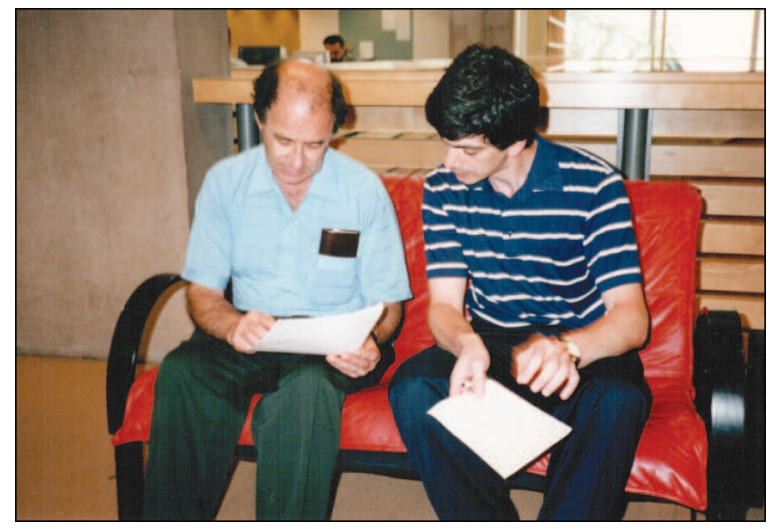

V. Arnold and B. Khesin, Toronto, 1997.

\section{Boris Khesin}

\section{On V. I. Arnold and Hydrodynamics}

Back in the mid-1980s, Vladimir Igorevich once told us, his students, how different the notion of "being young" (and in particular, being a young mathematician) is in different societies. For instance, the Moscow Mathematical Society awards an annual prize to a young mathematician under thirty years of age. The Fields Medal, as is well known, recognizes outstanding young mathematicians whose age does not exceed forty in the year of the International Congress. Both of the above requirements are strictly enforced.

This can be compared with the Bourbaki group, which is comprised of young French mathematicians and which, reportedly, has an age bar of fifty. However, as Arnold elaborated the story, this limit is more flexible: upon reaching this age the Bourbaki member undergoes a "coconutization procedure". The term is derived from a tradition of some barbaric tribe that allows its chief to carry out his duties until someone doubts his leadership abilities. Once the doubt arises, the chief is forced to climb to the top of a tall palm tree, and the whole tribe starts shaking it. If the chief is strong enough to get a good grip and survives the challenge, he is allowed to climb down and continue to lead the tribe until the next "reasonable doubt" in his leadership crosses someone's mind. If his grip is weak and he falls down from the 20-meter-tall tree, he obviously needs to be replaced, and so the next tribe chief is chosen. This tree is usually a coconut palm, which gave the name to the coconutization procedure.

As far as the coconutization in the Bourbaki group is concerned, according to Arnold's story, the unsuspecting member who reaches fifty is

Boris Khesin is professor of mathematics at the University of Toronto. His email address is khesin@ math. toronto.edu. 
invited, as usual, to the next Bourbaki seminar. Somewhere in the middle of the talk, when most of the audience is already half asleep, the speaker, who is in on the game for that occasion, inserts some tedious half-a-page-long definition. It is at this very moment that the scrutinized ("coconutized") member is expected to interrupt the speaker by exclaiming something like, "But excuse me, only the empty set satisfies your definition!" If he does so, he has successfully passed the test and will remain a part of Bourbaki. If he misses this chance, nobody says a word, but he will probably not be invited to the meetings any longer.

Arnold finished this story by quoting someone's definition of youth in mathematics which he liked best: "A mathematician is young as long as he reads works other than his own!"

Soon after this "storytelling" occasion, Arnold's fiftieth anniversary was celebrated: in June 1987 his whole seminar went for a picnic in a suburb of Moscow. Among Arnold's presents were a "Return to Arnold" stamp to mark the reprints he gave to his students to work on, a mantle with a nicely decorated "swallowtail", one of low-dimensional singularities, and such. But, most importantly, he was presented with a poster containing a crossword on various notions from his many research domains. Most of the questions were rather intricate, which predictably did not prevent Arnold from easily cracking virtually everything. But one question remained unresolved: a five-letter word (in the English translation) for "A simple alternative of life". None of the ideas worked for quite some time. After a while, having made no progress on this question, Arnold pronounced sadly, "Now I myself have been coconutized...." But a second later he perked up, a bright mischievous expression on his face: "This is a PURSE!" (In addition to the pirate's alternative "Purse or Life", the crossword authors meant the term "purse" in singularity theory standing for the description of the bifurcation diagram of the real simple singularity $D_{4}^{+}$, also called hyperbolic umbilichence the hint on "simple" alternative.)

Arnold's interest in fluid dynamics can be traced back to his "younger years", whatever definition one is using for that purpose. His 1966 paper in the Annales de l'Institut Fourier had the effect of a bombshell. Now, over forty years later, virtually every paper related to the geometry of the hydrodynamical Euler equation or diffeomorphism groups cites Arnold's work on the starting pages. In the next four or five years Arnold laid out the foundations for the study of hydrodynamical stability and for the use of Hamiltonian methods there, described the topology of steady flows, etc.

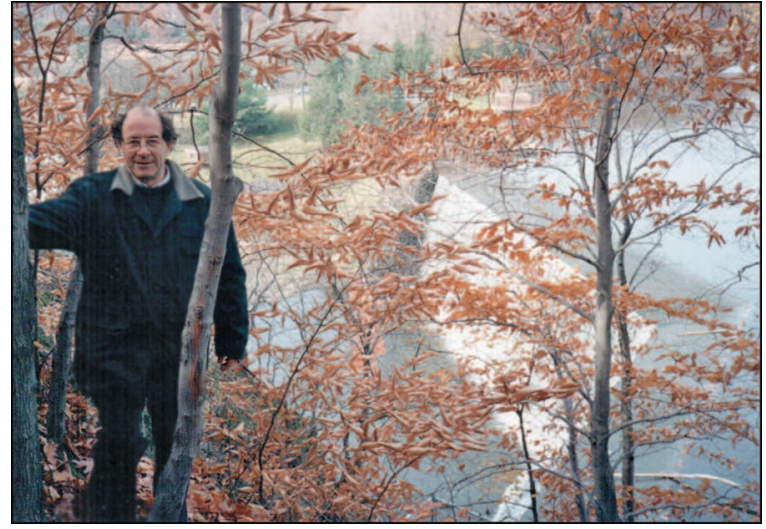

New Haven, 1993.

Apparently Arnold's interest in hydrodynamics is rooted in Kolmogorov's turbulence study and started with the program outlined by Kolmogorov for his seminar in 1958-59. Kolmogorov conjectured stochastization in dynamical systems related to hydrodynamical PDEs as viscosity vanishes, which would imply the practical impossibility of long-term weather forecasts. Arnold's take on hydrodynamics was, however, completely different from Kolmogorov's and involved groups and topology.

The Euler equation of an ideal incompressible fluid filling a domain $M$ in $\mathbb{R}^{n}$ is the evolution equation

$$
\partial_{t} v+(v, \nabla) v=-\nabla p
$$

on the fluid velocity field $v$, where this field is assumed to be divergence-free and tangent to the boundary of $M$ (while the pressure $p$ is defined uniquely modulo an additive constant by these conditions on $v$ ). In 1966 Arnold showed that this Euler equation can be regarded as the equation of the geodesic flow on the group $\operatorname{SDiff}(M)$ of volumepreserving diffeomorphisms of the domain $M$. The corresponding metric on this infinite-dimensional group is the right-invariant $L^{2}$ metric defined by the kinetic energy $E(v)=\frac{1}{2}\|v\|_{L^{2}(M)}^{2}$ of the fluid. (The analysis of Sobolev spaces related to this group-theoretic framework in incompressible fluid dynamics was later furnished by D. Ebin and J. Marsden.) Arnold's geometric view on hydrodynamics opened a multitude of different research directions:

Other groups and metrics. Many other evolution equations turned out to fit this universal approach suggested by Arnold, as they were found to describe geodesic flows on appropriate Lie groups with respect to one-sided invariant metrics. This shed new light on the corresponding configuration spaces and symmetries behind the relevant physical systems, and such geodesic equations are now 


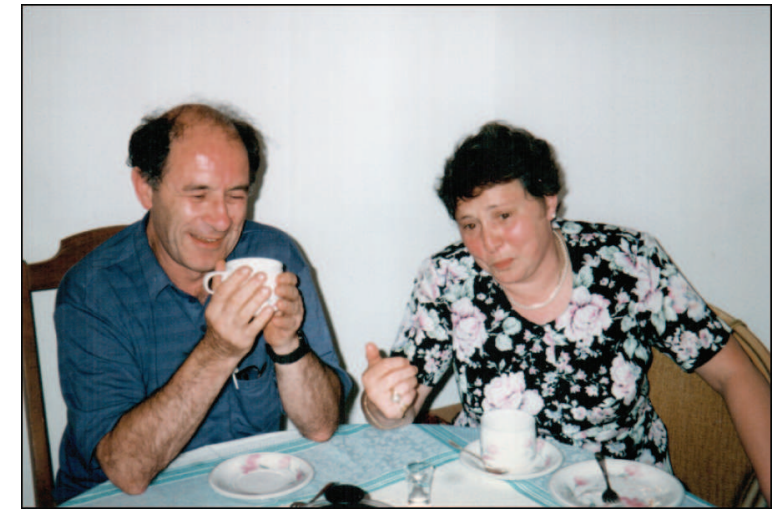

Vladimir Arnold with his wife, Elya, 1997.

called the Euler-Arnold equations. Here are several examples developed by many authors. The group $S O$ (3) with a left-invariant metric corresponds to the Euler top (this example appeared in the original paper by Arnold along with the hydrodynamical Euler equation). Similarly, the Kirchhoff equations for a rigid body dynamics in a fluid describe geodesics on the group $E(3)=S O(3) \ltimes \mathbb{R}^{3}$ of Euclidean motions of $\mathbb{R}^{3}$. In infinite dimensions, the group of circle diffeomorphisms $\operatorname{Diff}\left(S^{1}\right)$ with the right-invariant $L^{2}$-metric gives the inviscid Burgers equation, while the Virasoro group for three different metrics, $L^{2}, H^{1}$, and $\dot{H}^{1}$, produces respectively the Korteweg-de Vries, Camassa-Holm, and Hunter-Saxton equations, which are different integrable hydrodynamical approximations. The self-consistent magnetohydrodynamics describing simultaneous evolution of the fluid and magnetic field corresponds to dynamics on the semidirect product group $\operatorname{SDiff}(M) \ltimes \operatorname{SVect}(M)$ equipped with an $L^{2}$-type metric. Yet another interesting example, known as the Heisenberg chain or Landau-Lifschitz equation, corresponds to the gauge transformation group $C^{\infty}\left(S^{1}, S O(3)\right)$ and $H^{-1}$-type metric. Teasing physicists, Arnold used to say that their gauge groups are too simple to serve as a model for hydrodynamics.

Arnold's stability and Hamiltonian methods in hydrodynamics. The geodesic property of the Euler hydrodynamical equation implied that it is Hamiltonian when considered on the dual of the Lie algebra of divergence-free vector fields. Arnold proposed using the corresponding Casimir functions, which are invariants of the flow vorticity, to study stability of steady fluid flows. Arnold's stability is now the main tool in the study of nonlinear stability of fluid motions and MHD flows. In particular, he proved that planar parallel flows with no inflection points in their velocity profiles are stable. (One should note that, for Hamiltonian systems, stability in linear approximation is always neutral and inconclusive about the stability in the corresponding nonlinear problem, so the result on a genuine Lyapunov stability of certain fluid flows was particularly rare and valuable.)

Study of fluid Lagrangian instability and curvatures of diffeomorphism groups. Negative sectional curvature on manifolds implies exponential divergence of geodesics on them. In the 1966 Ann. Inst. Fourier paper Arnold launched the first computations of curvatures for diffeomorphism groups. Negativity of most of such curvatures for the groups of volume diffeomorphisms suggested Lagrangian instability of the corresponding fluid flows. By applying this to the the atmospheric flows, he gave a qualitative explanation of unreliability of long-term weather forecasts (thus answering in his own way the problem posed by Kolmogorov in the 1950s). In particular, Arnold estimated that, due to exponential divergence of geodesics, in order to predict the weather two months in advance one must have initial data on the state of the Earth's atmosphere with five more digits of accuracy than that of the expected prediction. In practical terms this means that a dynamical weather forecast for such a long period is impossible.

The hydrodynamical Appendix 2 in the famous Classical Mechanics by Arnold, ${ }^{8}$ where one can find the details of the above-mentioned calculation for the Earth's atmosphere, also contains one of Arnold's widely cited phrases: "We agree on a simplifying assumption that the earth has the shape of a torus," which is followed by his calculations for the group of area-preserving torus diffeomorphisms. It is remarkable that the later curvature calculations for the group of sphere diffeomorphisms (performed by A. Lukatskii) gave exactly the same order of magnitude and quantitative estimates for the curvature, and hence for the atmospheric flows, as Arnold's original computations for the torus!

Topology of steady flows. One of the most beautiful observations of Arnold (and one of the simplest -it could have belonged to $\mathrm{Eu}$ ler!) was the description of topology of stationary solutions of the 3D Euler equation. It turns out that for a "generic" steady solution the flow domain is fibered (away from a certain hypersurface) into invariant tori or annuli. The corresponding fluid motion on each torus is either periodic or quasiperiodic, while on each annulus it is periodic. This way a steady 3D flow looks like a completely

\footnotetext{
${ }^{8}$ Speaking of writing, once I asked Arnold how he managed to make his books so easy to read. He replied: "To make sure that your books are read fast, you have to write them fast." His own writing speed was legendary. His book on invariants of plane curves in the AMS University Lecture series was reportedly written in less than two days. Once he pretended to complain: "I tried, but failed, to write more than 30 pages a day...I mean to write in English; of course, in Russian, I can write much more!"
} 
integrable Hamiltonian system with two degrees of freedom.

The nongeneric steady flows include Beltrami fields (those collinear with their vorticity) and, in particular, the eigenfields for the curl operator on manifolds. The latter include the so-called ABC flows (for Arnold-Beltrami-Childress), the curl eigenfields on the 3D torus, which happen to have become a great model for various fast dynamo constructions.

Fast dynamo and magnetohydrodymanics. Arnold's interest in magnetohydrodynamics was to a large extent related to his acquaintance with Ya. Zeldovich and A. Sakharov. One of the results of their interaction at the seminars was the Arnold-Ruzmaikin-Sokolov-Zeldovich model of the fast dynamo on a 3D Riemannian manifold constructed from Arnold's cat map on a 2D torus. For a long time this was the only dynamo construction allowing complete analytical study for both zero and positive magnetic dissipation.

The asymptotic Hopf invariant. Finally, one of the gems of topological hydrodynamics is Arnold's 1974 study of the asymptotic Hopf invariant for a vector field. He proved that, for a divergence-free vector field $v$ in a $3 \mathrm{D}$ simply connected manifold $M$, the field's helicity, $H(v):=\int_{M}\left(c u r l^{-1} v, v\right) d^{3} x$, is equal to the average linking number of all pairs of trajectories of $v$. This theorem simultaneously generalized the Hopf invariant from maps $S^{3} \rightarrow S^{2}$ to arbitrary divergence-free vector fields in $S^{3}$, enriched K. Moffatt's result on the helicity of linked solid tori, described the topology behind the conservation law of the 3D Euler equation, and provided the topological obstruction to the energy relaxation of magnetic vector fields. This elegant theorem stimulated a tide of generalizations to higher-dimensional manifolds, to linking of foliations, to higher linkings, and to energy estimates via crossing numbers. In particular, there was substantial progress in the two directions suggested in the original 1974 paper: the topological invariance of the asymptotic Hopf numbers for a large class of systems was proved by J.-M. Gambaudo and É. Ghys, while the Sakharov-Zeldovich problem on whether one can make arbitrarily small the energy of the rotation field in a 3D ball by a volume-preserving diffeomorphism action was affirmatively solved by M. Freedman.

Virtually single-handedly Arnold spawned a new domain, now called topological fluid dynamics. His contribution to this area changed the whole paradigm of theoretical hydrodynamics by employing groups to study fluid flows. What doubles the awe is that this gem appeared almost at the same time with two other Arnold's foundational contributions-the KAM and singularity theories.

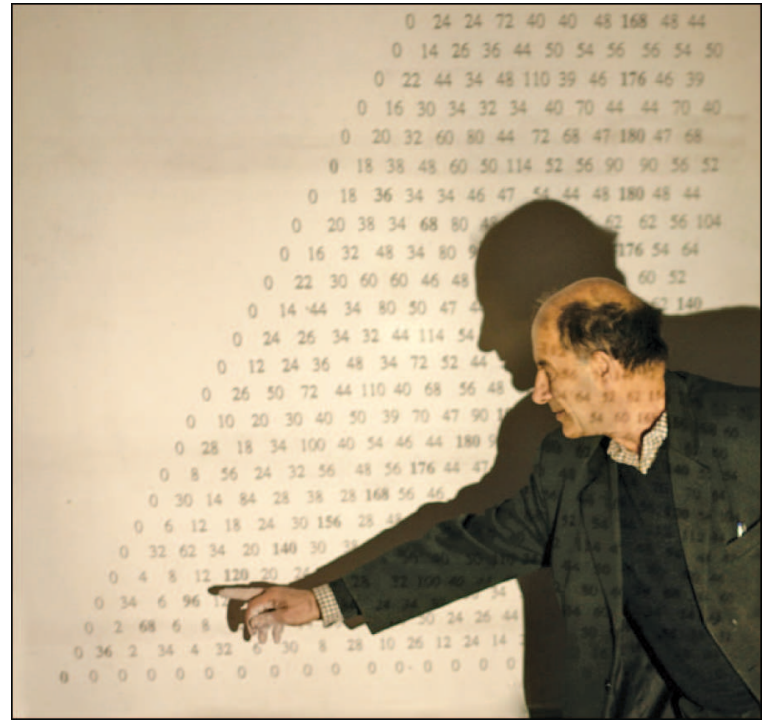

Vladimir Arnold lecturing.

\section{Victor Vassiliev}

\section{Topology in Arnold's Work}

Arnold worked comparatively little on topology for topology's sake. His topological studies were usually motivated by specific problems from other areas of mathematics and physics: algebraic geometry, dynamical systems, symplectic geometry, hydrodynamics, geometric and quantum optics. So the (very significant) place of topological studies in his work is well balanced with the (equally very significant) place and applications of topology in the entirety of contemporary mathematics.

The main achievement in a number of his works is a proper recognition and formulation of a topological result, allowing topologists to enter the area with their strong methods. A huge part of Arnold's work is contained not in his own articles but in well-formulated problems and hints that he gave to his students and other researchers; see especially [13]. So I will discuss below such Arnold hints as well and what followed from them.

\section{Superpositions of Functions}

The case of real functions: Kolmogorov-Arnold's theorem and Hilbert's 13th problem. This theorem states that every continuous function of $n>2$ variables can be represented by a superposition of functions in 2 variables (and the superposition can be taken in a particular form). The first approach to this problem (based on the notion of the Kronrod tree of connected components of level sets) was found by Kolmogorov (1956),

Victor Vassiliev is professor of mathematics at the Steklov Institute of Mathematics Higher School of Economics and at the Independent University of Moscow. His email address is vva@mi . ras. ru. 


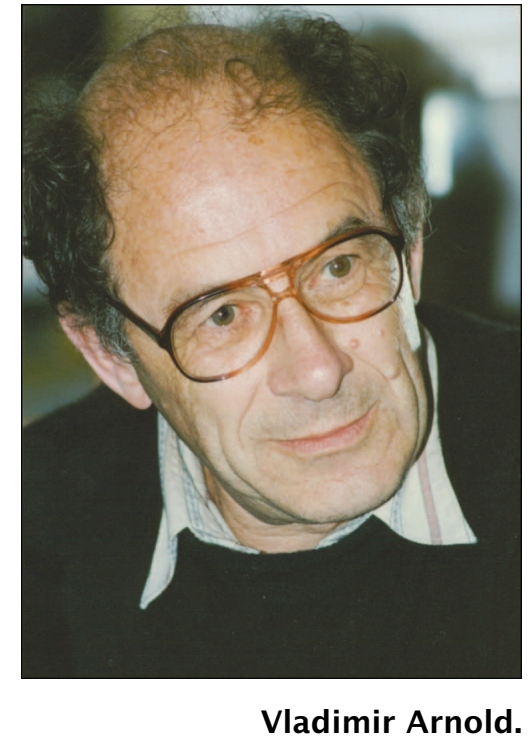

who did not, however, overcome some technical low-dimensional difficulties and proved only the same theorem with 2 replaced by 3 . The final effort was made by (then-19-year-old) Arnold.

This theorem gives a negative solution to (probably the most natural exact understanding of) the following Hilbert 13th problem:

...it is probable that the root of the equation of the seventh degree is a function of its coefficients which does not belong to this class of functions capable of nomographic construction, i.e., that it cannot be constructed by a finite number of insertions of functions of two arguments. In order to prove this, the proof would be necessary that the equation of the seventh degree

$$
t^{7}+x t^{3}+y t^{2}+z t+1=0
$$

is not solvable with the help of any continuous functions of only two arguments.

A widespread belief concerning this problem is as follows: "with the help of functions" in its last sentence means that a continuous solution $t(x, y, z)$ of (1) should indeed be given by a function of the form described in the first one, i.e., by a superposition of continuous functions of two arguments. In this case the Kolmogorov-Arnold theorem would give a direct negative answer to this problem. Nevertheless, this understanding of Hilbert's question is probably erroneous, because (1) does not define any continuous function at all: the multivalued function $t(x, y, z)$ defined by (1) does not have any continuous cross-section on the whole of $\mathbb{R}_{(x, y, z)}^{3}$. Indeed, such negative-valued cross-sections do not already exist in a small neighborhood of the polynomial

$$
\begin{aligned}
t^{7}- & 14 t^{3}-21 t^{2}-7 t+1 \\
& \equiv(t+1)^{3}\left(t^{4}-3 t^{3}+6 t^{2}-10 t+1\right) .
\end{aligned}
$$

Such a neighborhood admits two positive-valued cross-sections, but they obviously cannot be continued to the polynomial $t^{7}+1$. So this direct understanding of the Hilbert problem could be correct only under the (quite improbable) conjecture that Hilbert has included in this problem the question whether (or was confident that) (1) defines a continuous function on the entire $\mathbb{R}^{3}$; in this case the problem would have a positive solution.

A more realistic assumption is that "with the help of continuous functions of two variables" means something more flexible, for example, that we can consider a triple of functions $\left(\chi, g_{1}, g_{2}\right)$ in $x, y, z$, defined by such superpositions, and represent our function $t(x, y, z)$ by $g_{1}$ in the area where $x>0$ and by $g_{2}$ where $x \leq 0$. However, in this case it is unclear why Hilbert did not believe that the desired representation (maybe with more functions $\chi_{k}$ and $g_{i}$ ) does exist for his particular function, which is piecewise analytic and certainly can be stratified by easy conditions into pieces with very simple behavior. The most realistic conjecture is that (like for many other problems) Hilbert wrote a slightly obscure sentence specifically to let the readers themselves formulate (and solve) the most interesting and actual exact statements: it is exactly what Kolmogorov and Arnold actually did.

Complex algebraic functions and braid cohomology. Hilbert's 13th problem, formally asking something about real continuous functions, is nevertheless evidently motivated by the study of superpositions of multivalued algebraic functions in complex variables. A dream problem in this area is to solve literally the same problem concerning such functions. Moreover, this problem was explicitly formulated in one of Hilbert's consequent works.

Arnold worked much on this problem, revising and reformulating the proof of the Ruffini-Abel theorem in topological terms of ramified coverings and their topological invariants and trying to extend it to superpositions of functions in more variables. Although the exact desired theorem was not proved, a byproduct of this attack was huge: among other topics, it contains the topological theory of generalized discriminants, homological theory of braid groups, and theory of plane arrangements. A particular result, the topological obstruction to the representation by complete superpositions of functions depending on few variables, was expressed in [5] in the terms of 
cohomology of braid groups. Indeed, the $d$-valued algebraic function $t\left(x_{1}, \ldots, x_{d}\right)$ given by

$$
t^{d}+x_{1} t^{d-1}+\cdots+x_{d-1} t+x_{d}=0
$$

defines a $d$-fold covering over the set $\mathbb{C}^{d} \backslash \Sigma$ of nondiscriminant points $\left(x_{1}, \ldots, x_{d}\right)$ (i.e., of polynomials (2) for which all $d$ values $t(x)$ are different). This covering defines (up to homotopy) a map from its base $\mathbb{C}^{d} \backslash \Sigma$ to the classifying space $K(S(d), 1)$ of all $d$-fold coverings, thus also a canonical map

$$
H^{*}\left(K(S(d), 1) \rightarrow H^{*}\left(\mathbb{C}^{d} \backslash \Sigma\right) .\right.
$$

If our algebraic function (2) is induced from another one, as in the definition of complete superpositions, then this cohomology map factorizes through the cohomology ring of some subset of the argument space of this new algebraic function. Hence the dimension of this space cannot be smaller than the highest dimension in which the map (3) is nontrivial.

This approach has strongly motivated the study of the cohomology ring of the space $\mathbb{C}^{d} \backslash \Sigma$ (which is the classifying space of the d-braid group) and, much more generally, of the following objects.

\section{Discriminants and Their Complements}

Given a space of geometric objects (say, functions, varieties, subvarieties, matrices, algebras, etc.), the discriminant subset in it consists of all degenerate (in some precise sense) objects: it may be the set of non-Morse functions or selfintersecting spatial curves, or degenerate (another version: having multiple eigenvalues) operators. Usually one studies the complementary space of nonsingular objects. However, Arnold's seminal reduction replaces the homological part of this study by that of discriminant spaces. Namely, in [4], Arnold exploits the Alexander isomorphism

$$
H^{i}\left(\mathbb{C}^{d} \backslash \Sigma\right) \equiv \bar{H}_{2 d-i-1}(\Sigma),
$$

where $\bar{H}_{*}$ means the homology of the one-point compactification and $\mathbb{C}^{d}$ is considered to be the space of all complex polynomials (2) in one variable $t$. This reduction turned out to be extremely fruitful, because the set of nonsingular objects is usually open and does not carry any natural geometric structure. To study its topology, we often need to introduce some artificial structures on it, such as Morse functions, connections, families of vector fields or plane distributions, etc., which can have singularities helping us to calculate some topological invariants. On the other hand, the discriminant varieties are genuinely stratified sets (whose stratification corresponds to the hierarchy of singularity types); this stratification allows one to calculate various topological properties of these varieties and hence also of their complementary sets of generic objects. Already in [4] this approach has brought some progress, although the complete calculation of the group (4) was done only later by

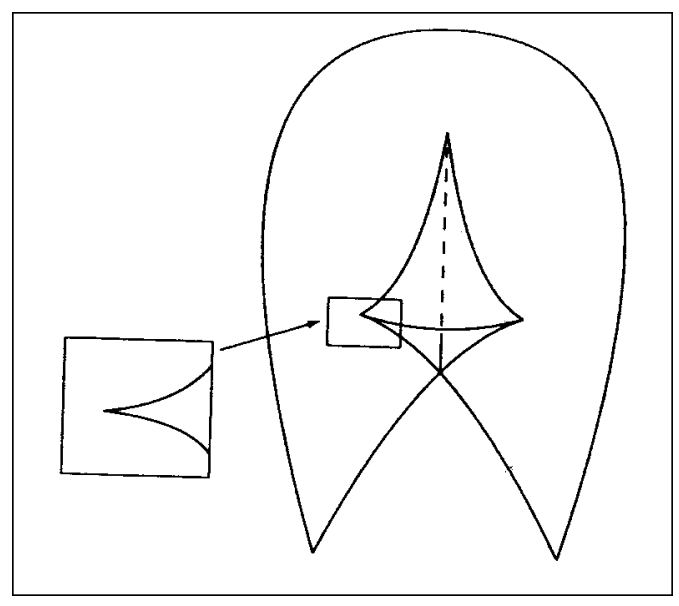

Figure 1. Stabilization of unfoldings.

D. Fuchs for $\mathbb{Z}_{2}$-cohomology [14] and by F. Cohen and F. Vainshtein for integral cohomology.

Using the same approach, Arnold studied later many other spaces of nondegenerate objects, namely, spaces $P_{d} \backslash \Sigma_{k}$ of real degree $d$ polynomials $\mathbb{R}^{1} \rightarrow \mathbb{R}^{1}$ without roots of multiplicity $\geq k$, $k \geq 3$, spaces of functions $\mathbb{R}^{1} \rightarrow \mathbb{R}^{1}$ (with a fixed behavior at infinity) also having no zeros of multiplicity $\geq k$ (1989), spaces of Hermitian operators with simple spectra (1995), spaces of generic (or generic Legendrian) plane curves (1994), etc.

Another very important idea of Arnold's in this area was his favorite stabilization problem, published first in 1976 and repeated many times in seminars; see problems 1975-19, 1980-15, 1985-7, 1985-22 in [13]. Formally speaking, the Alexander duality theorem is a finite-dimensional result. Also, all spaces of objects in which Arnold's approach originally led to more or less explicit results were finite-dimensional spaces considered as unfoldings of some particular objects. For example, the space $\mathbb{C}^{d}$ of complex polynomials (2) can be considered as an unfolding of the monomial $t^{d}$. When the degree $d$ grows, the cohomology groups of spaces $\mathbb{C}^{d} \backslash \Sigma$ of nondiscriminant polynomials stabilize (to the cohomology of the infinite braid group), but it was quite difficult to trace the stabilization process in terms of the original calculations. Moreover, it was unclear what happens with similar stabilizations for objects more complex than just polynomials in one variable, how to deal with similar infinite-dimensional problems, and what is "the mother of all unfoldings". To attack this set of philosophical problems, Arnold formulated a very explicit sample problem. First, he noticed that the stabilization of cohomology groups such as (2) is natural: if we have two singular objects, one of which is "more singular" than the other, then the parameter space of the unfolding of the simpler object can be embedded into that of the more complicated one. This map sends one discriminant into the other, thus inducing the pull-back 


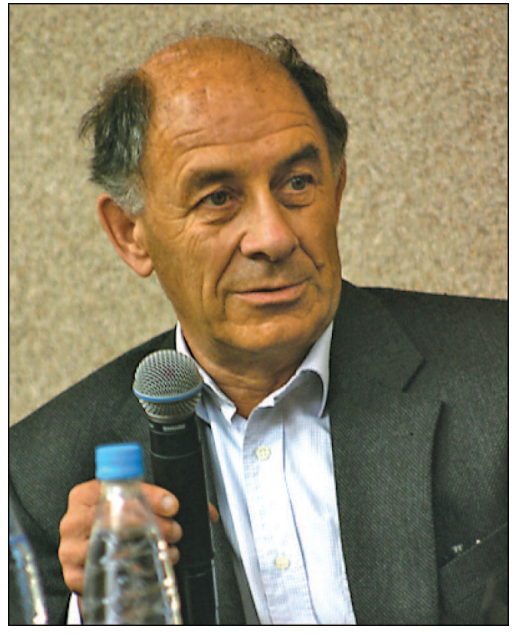

Vladimir Igorevich Arnold.

map of cohomology groups of their complements. (For real polynomials $t^{3}$ and $t^{4}$ this embedding of parameter spaces of their unfoldings $t^{3}+a t+b$ and $t^{4}+\alpha t^{2}+\beta t+\gamma$ is shown in Figure 1. The discriminants drawn in this picture are the sets of polynomials having multiple roots.)

Arnold's respective problem was to determine the stable (under all such pull-back maps) cohomology groups of such complements of discriminants of isolated singularities of holomorphic functions in $\mathbb{C}^{n}$ (and to prove that they actually do stabilize; i.e., these stable cohomology groups are realized by such groups for some sufficiently complicated singularities). Solving this problem, I found in 1985 a method of calculating homology groups of discriminants that behaves nicely under the embeddings of unfoldings and thus gives an effective calculation of stable groups. Some elaborations and byproducts of this calculation method constitute a majority of my results on topology of discriminants, including my first works on knot theory. In the original problem on stable cohomology of complements of discriminants of holomorphic functions, this calculation gives us the following formula: the desired stable cohomology ring for singularities in $n$ complex variables is equal to $H^{*}\left(\Omega^{2 n} S^{2 n+1}\right)$, where $\Omega^{k}$ is the $k$-fold loop space.

Moreover, this Arnold problem not only dealt with the stabilization of particular finitedimensional objects, but it also gave an approach to the study of actual infinite-dimensional function spaces.

\section{Topology of Pure Braid Groups and Plane Arrangements}

Together with the cohomology of the usual braid groups (2), Arnold also investigated the pure braid group, i.e., the fundamental group of the set of ordered collections of $d$ distinct points in $\mathbb{C}^{1}$. The classifying space of this group is just the space $\mathbb{C}^{d}$ with all diagonal hyperplanes $\left\{x_{i}=x_{j}\right.$ for $\left.i \neq j\right\}$ removed. Arnold's calculation of its cohomology group [2] became a sample and a starting point of numerous generalizations and initiated the socalled theory of plane arrangements. The Arnold identity

$$
\omega_{i j} \wedge \omega_{j k}+\omega_{j k} \wedge \omega_{k i}+\omega_{k i} \wedge \omega_{i j}=0
$$

for basic classes of this cohomology ring later became one of the main ingredients of Kontsevich's construction of the universal finite-type knot invariant.

\section{Maslov Index, Lagrange and Legendre Cobor- dism}

Lagrange manifolds are specific $n$-dimensional submanifolds of the symplectic space $\mathbb{R}^{2 n}$ (or, more generally, of the cotangent bundle of an arbitrary manifold $M^{n}$ ). They occur in problems of geometric optics as the manifolds into which all rays of light considered in such a problem can be lifted without intersections, and in quantum optics as a first step in obtaining an asymptotic approximation of light diffusion. However, further steps of this asymptotic description impose some consistency condition: the composition of transition functions relating their expressions in neighboring local charts should define the identity operator when we go along a closed chain of such charts. This condition is best formulated in terms of a certain 1-cohomology class of the Lagrange manifold, its Maslov index. If the Lagrange manifold $L^{n} \subset T^{*} \mathbb{R}^{n}$ is generic, then this index can be defined as the intersection index with the singular locus of the projection $L^{n} \rightarrow \mathbb{R}^{n}$ to the "physical" configuration space. It is important for this definition that, for generic Lagrangian manifolds, this locus has a welldefined transversal orientation (so that crossing it, we can always say whether we are going to the positive or the negative side) and its singular points form a subset of dimension at most $n-3$ in $L^{n}$ (so that all homologous curves have one and the same Maslov index). If $L^{n}$ is orientable, then this index is even; the above self-consistency condition requires that the value of this index on any closed curve should be a multiple of 4 . Arnold [1] related this index with the topology of the Lagrange Grassmann manifold of all Lagrangian planes in the symplectic $\mathbb{R}^{2 n}$-space, i.e., of all planes that can be tangent to some Lagrange submanifolds in this space. This settles immediately various problems related to the invariance of the definition of the Maslov index, as well as to its stability under deformations of the Lagrange manifold.

In 1980 Arnold initiated the theory of Lagrange and Legendre cobordisms [11]. Light distribution in the area defines light distribution on its border: for instance, the reflected light on the wall is defined by the light in the entire room. This means that a Lagrange manifold in the cotangent bundle 


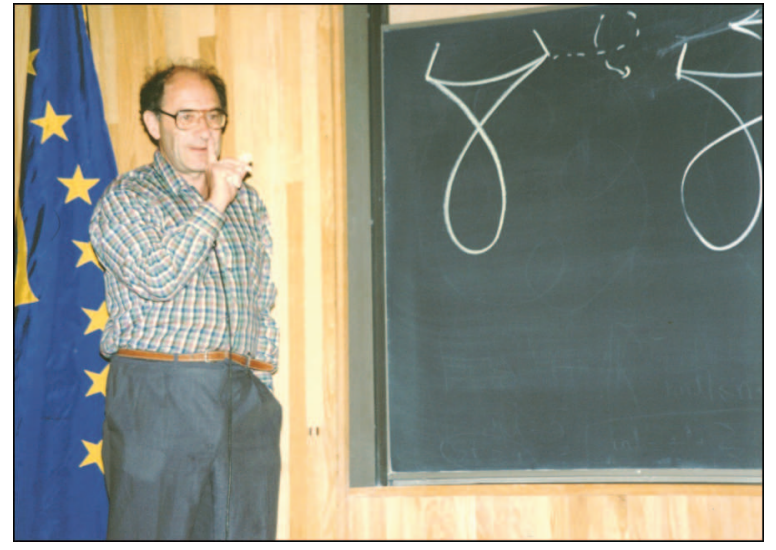

The Bowen lectures, Berkeley, 1997.

of the room defines its Lagrange boundary, which is a Lagrange manifold in the cotangent bundle of the wall. The Legendre manifolds are known to us mainly as resolutions of wave fronts. The wave front evolving in space defines a wave front of bigger dimension in the space-time. The fronts in $M^{n}$ corresponding to some instants $T_{1}$ and $T_{2}$ are obviously defined by the big front in $M^{n} \times\left[T_{1}, T_{2}\right]$; the way in which they are obtained from this big front can be generalized to the notion of the Legendre boundary. Notice that both Lagrange and Legendre boundaries of manifolds are not their boundaries and not even the subsets in the usual sense: they are obtained from these boundaries by symplectic and contact reductions.

Arnold introduced cobordism theories based on these boundary notions and calculated the 1-dimensional Lagrange and Legendre cobordism groups: they turned out to be isomorphic to $\mathbb{Z} \oplus \mathbb{R}$ and $\mathbb{Z}$, respectively. The $\mathbb{Z}$-term in both answers is defined by the Maslov index, the $\mathbb{R}$-invariant of the Lagrange cobordism is given by $\int p d q$. Later, Ya. Eliashberg and M. Audin, using the GromovLees version of the Smale-Hirsch $h$-principle for Lagrange manifolds, reduced the calculation of Legendre cobordism groups in any dimension to the standard objects of the cobordism theory, namely, to homotopy groups of appropriate Thom spaces (over the stable Lagrange Grassmann manifold).

At the same time, in the beginning of 1980, Arnold asked me whether it is possible to extend the construction of the Maslov index to cohomology classes of higher dimensions, dual to more degenerate singular loci of the Lagrangian projection $L^{n} \rightarrow \mathbb{R}^{n}$ than just the entire singular set. The resulting cohomology classes were expected to be closely related to the higher cohomology classes of Lagrange Grassmannians and to give invariants of Lagrange and Legendre cobordisms. The answer was found soon: I managed to construct the desired characteristic classes in terms of the universal complex of singularity types. Later, this theory was nicely and strongly extended by M. Kazarian in terms of equivariant homology.

On the other hand, the work with 1-dimensional wave fronts led Arnold to many essential problems of contact geometry, such as the 4-cusps problem (see the photograph above). Solutions of these problems by Chekanov, Eliashberg, Pushkar'and others resulted in significant development of this area.

There are many other topological results in Arnold's works, including major breakthroughs in real algebraic geometry [7], [10]; Arnold's conjecture in symplectic topology; the asymptotic Hopf invariant; and the vanishing homology theory of boundary singularities. These topics are covered in other articles in this collection.

\section{Helmut Hofer}

\section{Arnold and Symplectic Geometry}

V. I. Arnold was a character and a larger-than-life figure. I never knew him extremely well, but we became closer over the years, and I learned to know him a little bit more from the private side. He could be very charming.

As a student I read Arnold's wonderful book Mathematical Methods of Classical Mechanics and was impressed by the ease with which he was able to bring across important ideas. I never expected to meet him in real life.

I met him for the first time when I was a tenuretrack professor at Rutgers University and was visiting the Courant Institute. This was between 1986 and 1987, so around three years before the Berlin Wall and the iron curtain came down. The Courant Institute had worked hard to make it possible for Arnold to visit. I attended one of Arnold's lectures, which was remarkable in two ways: there was great mathematics and something one would not expect in a mathematics lecture. At some point he went into a tirade about how Western mathematicians were not giving proper credit to Russian mathematicians. Most people in the audience took it with some kind of amusement, but not all. Somebody sitting beside me mumbled something along the lines that we should have left him in Moscow.

A year or so later he attended parts of the symplectic year (1988) at MSRI in Berkeley. What I remember from his visit was that at some point he decided to swim in San Francisco Bay. One has to know that the locals do not consider this the best idea, since the currents are quite unpredictable. The story which was told at that time was that he almost drowned fighting the

Helmut Hofer is professor of mathematics at the Institute for Advanced Study. His email address is hofer@ias.edu. 
currents. I thought to myself, "That is a really interesting multidimensional character pushing the envelope." I recently asked about this of Richard Montgomery, who had an account of this story from Arnold himself. He had concluded from the description that Arnold had tried to swim from Marina Green to Marin (linked by the Golden Gate Bridge) during ebb tide and at some point, in his own words, "It felt like I hit a wall of current" and "had to turn back." The maximum ebb out of the San Francisco Bay can be over six knots. If he hadn't returned, he would have been swept at least a mile out to sea. Talking to Richard I also learned about another story. He and Arnold went kayaking in the bay. After an involuntary Eskimo roll, Arnold insisted on entering orthogonally into the path of an ongoing yacht race, with 40-foot yachts going full speed being unable to dodge a kayak. Richard still remembers his fear of going down in mathematical infamy as the guy who killed Arnold. As I said before, Arnold pushed the envelope in real life as he did in mathematics.

One year later, in 1989, I became a full professor at the Ruhr-Universität Bochum. Shortly afterwards the Berlin Wall came down, with dramatic changes in Eastern Europe. Soon a complete brain drain of the Soviet Union became a concern, and one day I found myself, together with my colleagues A. Huckleberry and V. Arnold, presiding over some research funding to allow Russian mathematicians to spend longer periods with a decent pay at Bochum. Arnold was very concerned, and I got to know him somewhat better. Professor Arnold became Dima.

Around 1994 I met him again; this time in Stanford. Dima, Yasha (Eliashberg), and I went looking for walnuts at the San Andreas Fault. I am sure it was Dima's idea. Knowing the "almost drowning version" of Dima's swimming expedition in San Francisco Bay, I had quite high expectations for the afternoon. However, there was no earthquake.

Around this point we started talking about mathematics, specifically symplectic topology. His opening bid was, "Helmut, you are using the wrong methods," referring to pseudoholomorphic curves, and I responded with, "I am sure you know something better. Make my day!" He liked to probe and enjoyed seeing people's reactions. I think I did well that day.

In 1998 he introduced my plenary lecture at the ICM in Berlin, and we had a friendly chat before the talk. The year before I had moved to the Courant Institute. He said, "Helmut, you should come back to Europe." I answered, "No, Dima, I love New York. But if it makes you feel better, consider me the agent of European culture in the U.S." I saw immediately that he liked this sentence. We talked about some more things which I rather thought would stay between us. Of course, I should have known better! He made it all part of his introduction and started by introducing me as the agent of European culture in the U.S., to the delight of many, but that was only the beginning; the rest is on video.

Dima had an amazing mathematical intuition and (which at this point shouldn't come as a surprise) was daring enough to make conjectures when others would not dare to stick their necks out.

There are quite a number of Arnold conjectures in symplectic geometry. However, there is one which even people outside of the field know and which was the initial driving force behind the development of symplectic geometry.

Arnold and Weinstein developed the modern language of symplectic geometry. This could, for example, be used to prove interesting perturbation results. However, there were no global results. Arnold was the one who raised these types of questions, and the Arnold conjecture I describe below is an example. Surprisingly, the breakthrough due to Conley and Zehnder came from outside the field.

In the following, I try to motivate the Arnold Conjecture. One can understand it as an analogy of the relationships between the Euler characteristic, the Hopf index formula, and the Lefschetz fixed point theorem. I haven't seen anything in his writings pointing out this analogy, but added it here as an intermediate step which helps to understand the conjecture better. Arnold describes a way of reasoning in Appendix 9 of his previously mentioned book. The Poincaré twist theorem can be seen as a special case of the two-dimensional torus case of his conjecture. The general case would be the generalization of the torus case to arbitrary closed symplectic manifolds. There is quite often a difference between the original thought process and the didactical cleaned version. From that point of view I regret that I never asked him how he arrived at his conjecture. The discussion below adds another point of view, constructing an analogy to a reasoning in topology. I very much believe that Arnold was aware of this analogy.

We start with a closed oriented manifold $M$ and a vector field $X$. The Euler characteristic $\chi(M)$ is a classical topological invariant, which is a generalization of the original concept introduced for polyhedra by Euler and which was fully generalized later by Poincaré. If $M$ is a smooth manifold, Hopf's index formula establishes a relationship between the zeros of a vector field assumed to be transversal to the zero section and the Euler characteristic of $M$ :

$$
\chi(M)=\sum_{m} i(X, m),
$$

where $i(X, m)= \pm 1$ is the local index at a zero of $X$. 


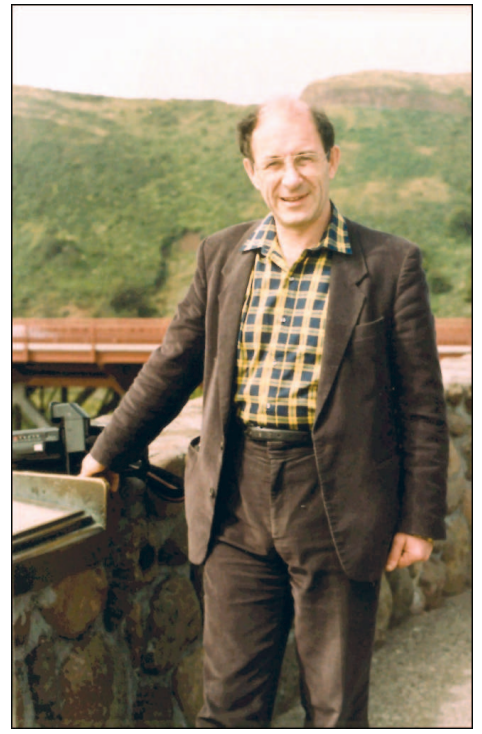

In Yosemite, California, 1989.

How can we generalize this? First we observe that a diffeomorphism can be viewed as a generalization of a vector field. Indeed, the collection of smooth vector fields can be viewed as the Lie algebra of the (Fréchet)-Lie group Diff $(M)$, so as an infinitesimal version of the latter. It is, however, not true that the diffeomorphisms close to the identity are in the image of the group exponential map. This is a consequence of being only a Fréchet Lie group and a universal problem in dealing with various sorts of diffeomorphism groups. Let us make a conjecture, which will come out as first going from the infinitesimal to the local to gain some confidence. We fix as an auxiliary structure a Riemannian metric with associated Riemannian exponential map exp. Assume that $\Phi$ is a diffeomorphism which is close to the identity. Then we can write $\Phi$ in a unique way in the form

$$
\Phi(m)=\exp _{m}(X(m)),
$$

for a small vector field $X$. Tranversality of $X$ to the zero-section is equivalent to $\Phi$ not having 1 in the spectrum of its linearizations at fixed points. Most importantly, the fixed points for $\Phi$ correspond to the zeros for $X$. Hence a generic diffeomorphism which is close to the identity has an algebraic fixed point count $\chi(M)$, where the sign is taken according to $\Phi^{\prime}(m)$ being orientation preserving or not. We can now make the "daring conjecture" that this should hold for all generic diffeomorphims isotopic to the identity. That turns out to be correct and is, of course, a special case of the Lefschetz fixed point formula.

What Arnold did in symplectic geometry is such a daring conjecture in a more complicated context. We start with a closed symplectic manifold $(M, \omega)$, and in analogy to the previous discussion we generalize the theory of functions on $M$ rather than the theory of vector fields. If $f$ is a smooth function with all critical points nondegenerate, then Morse theory says its number of critical points is at least the sum of the Betti numbers (for any coefficient field). Morse theory also tells us that the algebraic count of critical points is $\chi(M)$. Since we have a symplectic structure, we can associate to $f$ a vector field $X_{f}$ by the obvious formula

$$
d f=i_{X_{f}} \omega .
$$

This is the so-called Hamiltonian vector field. Obviously we are now back to the first discussion. However, with the vector fields being more special, one would like a stronger statement for a certain class of diffeomorphisms. This particular class of diffeomorphisms should generalize functions as diffeomorphisms isotopic to the identity generalize vector fields.

Symplectic diffeomorphisms isotopic to the identity are not a good guess, since for $T^{2}$ with the standard symplectic form a small translation would give no fixed points at all. We could, however, look at all symplectic diffeomorphisms obtained as time- 1 maps for the family of vector fields $X_{f_{t}}$ for a smooth time-dependent family $f:[0,1] \times M \rightarrow \mathbb{R}$, with $f_{t}(x):=f(t, x)$. This produces the group of all Hamiltonian diffeomorphisms $\operatorname{Ham}(M, \omega)$. Indeed the collection of smooth maps can be viewed as the Lie algebra for $\operatorname{Ham}(M, \omega)$.

How can we go from the infinitesimal to the local, as we did in the previous discussion? A basic and not too difficult symplectic result is that the neighborhood of a Lagrangian submanifold of a symplectic manifold is symplectically isomorphic to a neighborhood of the zero-section in its cotangent bundle with the natural symplectic structure. Now comes a little trick which replaces the use of the exponential map associated to an auxiliary metric. We define $N=M \times M$ with the form $\tau=\omega \oplus(-\omega)$. Then the diagonal $\Delta_{M}$ is a Lagrange submanifold of $N$, and an open neighborhood of it looks like an open neighborhood of $\Delta_{M}$ in $T^{*} \Delta_{M}$. Every symplectic map that is sufficiently close to the identity has a graph which when viewed as a subset of $T^{*} \Delta_{M}$ is a graph over the zero-section, i.e., the graph of a one-form $\lambda$. An easy computation shows that the original diffeomorphism is symplectic if and only if $\lambda$ is closed. It is Hamiltonian if and only if $\lambda$ is exact:

$$
\lambda=d g
$$

for some smooth function. Hence the fixed points of a Hamiltonian diffeomorphism $\Phi$ correspond to the intersection of its graph with the zerosection and hence with the critical points of $g$. Now we are in the local situation, similarly as in the previous case. We conclude that a generic element in $\operatorname{Ham}(M, \omega)$ has at least as many fixed points as a smooth function has critical points if it is close enough to the identity map. 
Knowing all this, Arnold makes the following daring conjecture (nondegenerate case, in my words).

Arnold Conjecture: A nondegenerate Hamiltonian diffeomorphism has at least as many fixed points as a Morse function has critical points.

It wouldn't be Dima if it actually was that straightforward. The most prominent statement "Arnold-style" of this conjecture is in his book Mathematical Methods of Classical Mechanics. In the Springer 1978 edition (being a translation of the 1974 Russian edition) it reads on page 419 (and this is a restatement of some published version of the conjecture in 1965):

Thus we come to the following generalization of Poincaré's theorem:

Theorem: Every symplectic diffeomorphism of a compact symplectic manifold, homologous to the identity, has at least as many fixed points as a smooth function on this manifold has critical points (at least if this diffeomorphism is not too far from the identity).

The symplectic community has been trying since 1965 to remove the parenthetical part of the statement. After tough times from 1965 to 1982, an enormously fruitful period started with the Conley-Zehnder theorem in 1982-83, proving the Arnold conjecture for the standard torus in any (even) dimension using Conley's index theory (a powerful version of variational methods). This was followed by Gromov's pseudoholomorphic curve theory coming from a quite different direction. At this point the highly flexible symplectic language becomes a real asset in the field. Finally, Floer combines the Conley-Zehnder viewpoint with that of Gromov, which is the starting point of Floer theory in 1987. As far as the Arnold conjecture is concerned, we understand so far a homological version of the nondegenerate case. A LuisternikShnirelman case (also conjectured by Arnold) is still wide open, though some partial results are known.

The development of symplectic geometry has been and still is a wonderful journey. Thanks, Dima!

\section{Acknowledgments}

The photographs are courtesy of the Arnold family archive, F. Aicardi, Ya. Eliashberg, E. Ferrand, B. and M. Khesin, J. Pöschel, M. Ratner, S. Tretyakova, and I. Zakharevich.

\section{References}

[1] V. ARNOLD, On a characteristic class participating in the quantization conditions, Funct. Anal. Appl. 1, no. 1 (1967), 1-14.

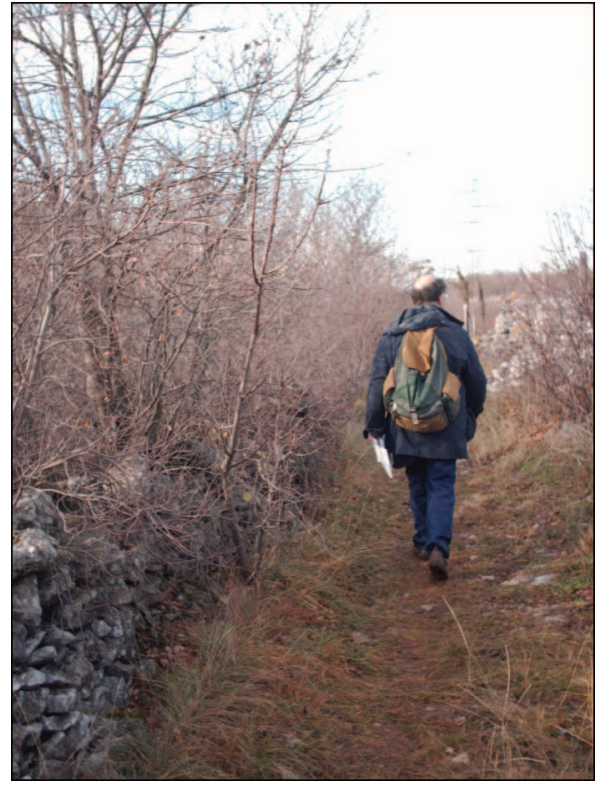

V. Arnold, 2008.

[2] _ , The cohomology ring of the group of colored braids, Mat. Zametki 5, no. 2 (1969), 227-231.

[3] — The one-dimensional cohomology of the Lie algebra of divergence-free vector fields, and the winding numbers of dynamical systems, Funct. Anal. Appl. 3, no. 4 (1969), 77-78.

[4] _ On some topological invariants of algebraic functions, Trudy Moskov. Matem. Obshch. 21 (1970), 27-46.

[5] , Topological invariants of algebraic functions. II, Funct. Anal. Appl. 4, no. 2 (1970), $1-9$.

[6] L_ Local problems of analysis, Vestnik Moskov. Univ. Ser. I Mat. Meh. 25 (1970), no. 2, 52-56.

[7] _ Distribution of ovals of real plane algebraic curves, involutions of 4-dimensional smooth manifolds, and arithmetics of integral quadratic forms, Funct. Anal. Appl. 5, no. 3 (1971), 1-9.

[8] _ Lectures on bifurcations and versal families, Russ. Math. Surveys 27 (1972), no. 5, 54-123.

[9] _ A spectral sequence for the reduction of functions to normal forms, Funct. Anal. Appl. 9 (1975), 81-82.

[10] _ Index of a singular point of a vector field, Petrovsky-Oleinik inequality, and mixed Hodge structures, Funct. Anal. Appl. 12, no. 1 (1978), 1-14.

[11] _ Lagrange and Legendre cobordisms, Funct. Anal. Appl. 14, no. 3 (1980), 1-13, and 14, no. 4 (1980), 8-17.

[12] _ Geometrical Methods in the Theory of Ordinary Differential Equations, Springer-Verlag, New York-Berlin, 1983.

[13] Arnold's Problems, Springer/Phasis, 2004.

[14] D. FuCHS, Cohomology of the braid group modulo 2, Funct. Anal. Appl. 4, no. 2 (1970), 62-73.

[15] H. WHITNEY, On singularities of mappings of Euclidean spaces. I, Mappings of the plane into the plane, Ann. of Math. (2) 62 (1955), 374-410. 